\title{
A self adaptive inertial subgradient extragradient algorithm for variational inequality and common fixed point of multivalued mappings in Hilbert spaces
}

https://doi.org/10.1515/dema-2019-0013

Received November 3, 2018; accepted January 28, 2019

Abstract: We consider a new subgradient extragradient iterative algorithm with inertial extrapolation for approximating a common solution of variational inequality problems and fixed point problems of a multivalued demicontractive mapping in a real Hilbert space. We established a strong convergence theorem for our proposed algorithm under some suitable conditions and without prior knowledge of the Lipschitz constant of the underlying operator. We present numerical examples to show that our proposed algorithm performs better than some recent existing algorithms in the literature.

Keywords: variational inequality, extragradient method, fixed point problem, accelerated algorithm, demicontractive mappings, viscosity approximation method

MSC: 49J40, 58E35, 65K15, 90C33

\section{Introduction}

Let $C$ be a nonempty, closed and convex subset of a real Hilbert space $H$ with inner product $\langle\cdot, \cdot\rangle$ and norm $\|\cdot\|$. The Variational Inequality Problem (VIP) is defined as finding a point $x^{\star} \in C$ satisfying

$$
\left\langle A x^{\star}, z-x^{\star}\right\rangle \geq 0, \text { for all } z \in C,
$$

where $A: C \rightarrow H$ is a given mapping. The VIP is an important tool in studying a wide class of problems arising in pure and applied science such as minimization problems, saddle point problems, partial differential equations, optimal control problems, economics, engineering and mathematical programming. We denote the set of solutions of the VIP by $\operatorname{VI}(C, A)$. This field is experiencing an explosive growth in both theory and applications. Several iterative methods have been developed for solving the VIP and its related optimization problems, see [1-5] and references therein. It is well known that the VIP is equivalent to the following fixed point problem (see [6])

$$
x^{\star}=P_{C}(I-\mu A) x^{\star},
$$

\footnotetext{
Lateef Olakunle Jolaoso: School of Mathematics, Statistics and Computer Science, University of KwaZulu-Natal, Durban, South Africa; E-mail: 216074984@stu.ukzn.ac.za, lateefjolaoso89@gmail.com

Adeolu Taiwo: School of Mathematics, Statistics and Computer Science, University of KwaZulu-Natal, Durban, South Africa; E-mail: 218086816@stu.ukzn.ac.za

Timilehin Opeyemi Alakoya: School of Mathematics, Statistics and Computer Science, University of KwaZulu-Natal, Durban, South Africa; E-mail: 218086823@stu.ukzn.ac.za, timimaths@gmail.com

*Corresponding Author: Oluwatosin Temitope Mewomo: School of Mathematics, Statistics and Computer Science, University of KwaZulu-Natal, Durban, South Africa; Email: mewomoo@ukzn.ac.za
} 
where $\mu>0$ is an arbitrary constant and $P_{C}$ is the metric projection from $H$ onto $C$. A well known algorithm for finding the solution of VIP is the extragradient method proposed by Korpelevich [7] which is given as follows:

Algorithm 1.1. (Extragradient Method (EgM))

$$
\left\{\begin{array}{l}
x_{0} \in C, \\
y_{n}=P_{C}\left(x_{n}-\mu A x_{n}\right), \\
x_{n+1}=P_{C}\left(x_{n}-\mu A y_{n}\right),
\end{array}\right.
$$

where $C \subseteq \mathbb{R}^{n}, A: C \rightarrow \mathbb{R}^{n}$ is a monotone and L-Lipschitz continuous operator with $\mu \in\left(0, \frac{1}{L}\right)$.

If the solution set $\operatorname{VI}(C, A)$ is nonempty, then the sequence $\left\{x_{n}\right\}$ generated by $E g M$ converges weakly to an element in $\operatorname{VI}(C, A)$. $\mathrm{n}$ recent years, the $E g M$ has been further extended to infinite dimensional spaces (see [8-12]). Note that the $E g M$ involves two projections onto the set $C$ and two evaluations of $A$ per iteration. A major improvement on the EgM is to minimize the number of evaluations of $P_{C}$ per iteration. An attempt in this direction was initiated by Y. Censor et al. [13] who modified the EgM method by replacing the second projection with a projection onto a half-space. This new method which thus involves only one projection onto $C$ is called the subgradient extragradient method and is given as follows:

Algorithm 1.2. (The Subgradient Extragradient method (SEgM))

$$
\left\{\begin{array}{l}
x_{0} \in H, \\
y_{n}=P_{C}\left(x_{n}-\mu A x_{n}\right), \\
Q_{n}=\left\{z \in H:\left\langle x_{n}-\mu A x_{n}-y_{n}, z-y_{n}\right\rangle \leq 0\right\}, \\
x_{n+1}=P_{Q_{n}}\left(x_{n}-\mu A y_{n}\right) .
\end{array}\right.
$$

Censor et al. [13] showed that if the solution set $\operatorname{VI}(C, A)$ is nonempty, the sequence $\left\{x_{n}\right\}$ generated by $S E g M$ converges weakly to an element $p \in V I(C, A)$, where $p=\lim _{n \rightarrow \infty} P_{V I(C, A)}\left(x_{n}\right)$. Using only a single projection onto $C$, Maingé and Gobinddass [14] (see also Maingé [15]) also obtained a result relating to weak convergence algorithm for approximating solutions of the VIP in a real Hilbert space by means of a projected reflected gradient-type method [16] and inertial terms. Several other alternatives to the EgM and its modification have also been proposed in the literature.

In solving optimization problems, strong convergence of iterative schemes are more desirable than their weak convergence counterparts as pointed out by Bauschke and Combettes in [17]. Therefore, it is beneficial to develop an algorithm that generates a strong convergent sequence.

Let $S$ be a nonlinear mapping and let $F(S)$ denote the set of fixed points of $S$ (i.e. $F(S):=\{x \in H: S x=x\}$ ). It is important to study the problem of finding a common solution of both the VIP and fixed point problem due to its possible applications to mathematical models whose constraints can be expressed as fixed point problems and variational inequalities. This happens, in particular, in practical problems such as in signal processing, network resource allocation, or image recovery, see for instance [18-25]. In [13], Y. Censor et al. studied the approximation of common solution to a variational inequality problem and fixed point problem for a nonexpansive mapping. In particular, they proposed the following $S E g M$ and proved its weak convergence to a solution $u^{\star} \in F(S) \cap V I(C, A)$ :

$$
\left\{\begin{array}{l}
x_{0} \in H, \mu>0, \\
y_{n}=P_{C}\left(x_{n}-\mu A x_{n}\right), \\
T_{n}=\left\langle z \in H:\left\langle x_{n}-\mu A x_{n}-y_{n}, z-y_{n}\right\rangle \leq 0\right\}, \\
x_{n+1}=\alpha_{n} x_{n}+\left(1-\alpha_{n}\right) S P_{T_{n}}\left(x_{n}-\mu A y_{n}\right) .
\end{array}\right.
$$

Motivated by the work of [26], Thong and Hieu [27] proposed the following two algorithms for finding a common element of the set of solutions of VIP and the fixed point of a demicontractive mapping. 
Algorithm 1.3. (THSEgM(I))

$$
\left\{\begin{array}{l}
x_{0} \in H \\
y_{n}=P_{C}\left(x_{n}-\mu A x_{n}\right), \\
T_{n}=\left\{x \in H:\left\langle x_{n}-\mu A x_{n}-y_{n}, x-y_{n}\right\rangle \leq 0\right\} \\
z_{n}=P_{T_{n}}\left(x_{n}-\mu A y_{n}\right) \\
x_{n+1}=\left(1-\alpha_{n}-\beta_{n}\right) z_{n}+\beta_{n} S z_{n}
\end{array}\right.
$$

Algorithm 1.4. (THSEgM(II))

$$
\left\{\begin{array}{l}
x_{0} \in H, \\
y_{n}=P_{C}\left(x_{n}-\mu A x_{n}\right), \\
T_{n}=\left\{x \in H:\left\langle x_{n}-\mu A x_{n}-y_{n}, x-y_{n}\right\rangle \leq 0\right\}, \\
z_{n}=P_{T_{n}}\left(x_{n}-\mu A y_{n}\right), \\
x_{n+1}=\left(1-\beta_{n}\right) \alpha_{n} z_{n}+\beta_{n} S z_{n},
\end{array}\right.
$$

where $S: H \rightarrow H$ is a $\lambda$-demicontractive mapping with $0 \leq \lambda<1$, and where $\left\{\alpha_{n}\right\},\left\{\beta_{n}\right\}$ are sequences in $(0,1)$. Under suitable conditions on the parameters $\alpha_{n}$ and $\beta_{n}$, they proved that the sequence $\left\{x_{n}\right\}$ generated by (5) and (6) converges strongly to a solution $p \in \operatorname{VI}(C, A) \cap F(S)$.

Also in 2000, Moudafi [28] introduced the viscosity approximation method for approximating fixed points of nonexpansive mappings. Let $f$ be a contraction on $H$, and start with an arbitrary $x_{0} \in H$. We then define a sequence $\left\{x_{n}\right\}$ recursively by

$$
x_{n+1}=\lambda_{n} f\left(x_{n}\right)+\left(1-\lambda_{n}\right) T x_{n}, \quad n \geq 0,
$$

where $\left\{\lambda_{n}\right\}$ is a sequence in $(0,1) . \mathrm{Xu}[29]$ proved that if $\left\{\lambda_{n}\right\}$ satisfy certain conditions, the sequence $\left\{x_{n}\right\}$ generated by (7) converges strongly to the unique solution $x \in F(T)$ of the variational inequality

$$
\left\langle(I-f) x^{\dagger}, x-x^{\dagger}\right\rangle \geq 0, \text { for all } x \in F(T) .
$$

Based on the heavy ball methods of a two-order time dynamical system, Polyak [30] first proposed an inertial extrapolation as an acceleration process to solve the smooth convex minimization problem. The inertial algorithm is a two-step iteration where the next iterate is defined by making use of the previous two iterates. Recently, several of researchers have constructed some fast iterative algorithms by using inertial extrapolation which includes inertial proximal methods [31, 32], inertial forward-backward methods [33], inertial split equilibrium methods [34], inertial proximal ADMM [35] and the fast iterative shrinkage thresholding algorithms FISTA [36, 37]. In 2008, using the technique of inertial extrapolation, Maingé [38] introduced the following inertial Mann algorithm:

$$
\left\{\begin{array}{l}
y_{n}=x_{n}+\theta_{n}\left(x_{n}-x_{n-1}\right), \\
x_{n+1}=\left(1-\lambda_{n}\right) y_{n}+\lambda_{n} T y_{n},
\end{array}\right.
$$

for each $n \geq 1$. He showed that the iterative sequence $\left\{x_{n}\right\}$ converges weakly to a fixed point of $T$ under the following conditions:

(A1) $\theta_{n} \in[0, \alpha)$ for each $n \geq 1$, where $\alpha \in[0,1)$,

(A2) $\quad \sum_{n=1}^{\infty} \theta_{n}\left\|x_{n}-x_{n-1}\right\|^{2}<+\infty$,

(A3) $0<\inf \lambda_{n} \leq \sup \lambda_{n}<1$.

To satisfy Condition (A2) of the sequence $\left\{x_{n}\right\}$, it is necessary to first calculate $\theta_{n}$ at each step (see [32]). In 2015, Bot and Csetnek [39] removed the condition (A2) and substituted (A1) and (A3) with the following conditions:

(B1) for each $n \geq 1,\left\{\theta_{n}\right\} \subset[0, \alpha)$ is nondecreasing with $\theta_{1}=0$ and $0 \leq \alpha<1$, 
(B2) for each $n \geq 1$,

$$
\delta>\frac{\alpha^{2}(1+\alpha)+\alpha \sigma}{1-\alpha^{2}}, \quad 0 \leq \lambda \leq \lambda_{n} \leq \frac{\delta-\alpha[\alpha(1+\alpha)+\alpha \delta+\sigma]}{\delta[1+\alpha(1+\alpha)+\alpha \delta+\sigma]}
$$

where $\lambda, \sigma, \delta>0$.

Recently, Dong et al. [40] introduced the following inertial extragradient algorithm by incorporating the inertial term in the EgA.

Algorithm 1.5. (Inertia Extragradient Algorithm (iEgA))

$$
\left\{\begin{array}{l}
w_{n}=x_{n}+\alpha_{n}\left(x_{n}-x_{n-1}\right), \\
y_{n}=P_{C}\left(w_{n}-\mu F\left(w_{n}\right)\right), \\
x_{n+1}=\left(1-\lambda_{n}\right) w_{n}+\lambda_{n} P_{C}\left(w_{n}-\mu F\left(y_{n}\right)\right),
\end{array}\right.
$$

where $\left\{\alpha_{n}\right\}$ is a nondecreasing sequence with $\alpha_{1}=0$ and $0 \leq \alpha_{n} \leq \alpha<1$ for any $n \geq 1$ and $\lambda, \sigma, \delta>0$ are such that

and

$$
\delta>\frac{\alpha\left[(1+\mu L)^{2} \alpha(1+\alpha)+\left(1-\mu^{2} L^{2}\right) \alpha \sigma+\sigma(1+\mu L)^{2}\right]}{1-\mu^{2} L^{2}}
$$

$$
0<\lambda_{n} \leq \frac{\delta\left(1-\mu^{2} L^{2}\right)-\alpha\left[(1+\mu L)^{2} \alpha(1+\alpha)+\left(1-\mu^{2} L^{2}\right) \alpha \sigma+\sigma\left(1+\mu L^{2}\right)\right]}{\delta\left[(1+\mu L)^{2} \alpha(1+\alpha)+\left(1-\mu^{2} L^{2}\right) \alpha \sigma+\sigma(1+\mu L)^{2}\right]} .
$$

Using the $i E g A$ (9), Dong et al. [40] proved a weak convergence result for an approximation of a solution of the VIP (1).

Observe that the stepsize $\mu$ of the extragradient, inertial extragradient and the subgradient extragradient algorithms play an essential role in the convergence properties of the iterative methods. The Lipschitz constant $L$ is typically assumed to be known, or at least estimated priorly. In many cases, this parameter is unknown or difficult to approximate. Moreover, the stepsize defined by this constant is often very small and deteriorates the convergence rate. In practice, a larger stepsize can often be used and yield better numerical results. It is thus natural to ask the following question:

Is it possible to have an inertial subgradient extragradient algorithm with self adaptive stepsize which converges strongly to a common solution of a variational inequality and fixed point problem?

It is our aim therefore to provide an affirmative answer to this question. Motivated by the work of Censor et al [26], Thong and Hieu [27] and Dong et al. [40], we introduce an inertial viscosity subgradient extragradient type algorithm with self adaptive stepsize. We also prove a strong convergence theorem for approximating a common solution of the VIP and a finite family of multivalued $\lambda$-demicontractive mappings in a real Hilbert space under some suitable conditions. Our algorithm is composed of a modified subgradient extragradient algorithm together with a viscosity approximation and inertial extrapolation. Stepsize is selected self adaptively, hence does not require a prior estimate of the Lipschitz constant $L$. Furthermore, we present some numerical examples to show that our proposed algorithm is more efficient than some existing ones in literature.

\section{Preliminaries}

Let $C$ be a nonempty, closed and convex subset of a real Hilbert space $H$. We denote the strong and weak convergence of a sequence $\left\{x_{n}\right\} \subseteq H$ to a point $p \in H$ by $x_{n} \rightarrow p$ and $x_{n} \rightarrow p$ respectively. For each $x \in H$, there exists a unique element $P_{C} x$ in $C$ such that

$$
\left\|P_{C}(x)-x\right\|=\min \{\|y-x\|: y \in C\} .
$$

The mapping $P_{C}$ is called the metric projection from $H$ onto $C$. It is well known that $P_{C}$ has the following characteristics: 
(i) $\left\langle x-y, P_{C} x-P_{C} y\right\rangle \geq\left\|P_{C} x-P_{C} y\right\|^{2}$, for every $x, y \in H$,

(ii) for $x \in H$ and $z \in C, z=P_{C} x$ if and only if

$$
\langle x-z, z-y\rangle \geq 0, \text { for all } y \in C,
$$

(iii) for $x \in H$ and $y \in C$,

$$
\left\|y-P_{C}(x)\right\|^{2}+\left\|x-P_{C}(x)\right\|^{2} \leq\|x-y\|^{2} .
$$

Given $x, y \in H, y \neq 0$, let $Q=\{z \in H:\langle y, z-x\rangle \leq 0\}$. Then, for all $u \in H$, the projection $P_{Q}(u)$ is defined by

$$
P_{Q}(u)=u-\max \left\{0, \frac{\langle y, u-x\rangle}{\|y\|^{2}}\right\} y,
$$

which gives us an explicit formula for finding the projection of any point onto a half space.

Definition 2.1. Let $A: H \rightarrow H$ be an operator. Then

(i) $A$ is called Lipschitz continuous with constant $L>0$ if

$$
\|A x-A y\| \leq L\|x-y\|, \quad \text { for all } x, y \in H,
$$

(ii) $A$ is called monotone if

$$
\langle A x-A y, x-y\rangle \geq 0, \quad \text { for all } x, y \in H .
$$

The normal cone of a nonempty closed convex subset $C$ of $H$ at a point $x \in C$, denoted by $N_{C}(x)$ is defined as

$$
N_{C}(x)=\{u \in H:\langle u, y-x\rangle \leq 0, \quad \text { for all } y \in C\} .
$$

Let $B: H \rightarrow 2^{H}$ be a multivalued operator on $H$. Then

(i) the graph $G(B)$ of $B$ is defined by

$$
G(B):=\{(x, u) \in H \times H: u \in B(x)\},
$$

(ii) the operator $B$ is called a maximal monotone operator if $B$ is monotone, i.e.

$$
\langle u-v, x-y\rangle \geq 0, \quad \text { for all } \quad u \in B(x), v \in B(y),
$$

and the graph $G(B)$ of $B$ is not properly contained in the graph of any other monotone operator.

It is clear that a monotone mapping $B$ is maximal if and only if for any $(x, u) \in H \times H$, if $\langle u-v, x-y\rangle \geq 0$ for all $(v, y) \in G(B)$, then $u \in B(x)$ (see [41], Theorem 3.3).

Let $A: C \rightarrow H$ be a monotone mapping and let $B: H \rightarrow 2^{H}$ be a mapping defined by

$$
B q= \begin{cases}A q+N_{C}(q), & q \in C, \\ \emptyset, & q \notin C .\end{cases}
$$

Then $B$ is maximal monotone and $x \in B^{-1}(0)$ if and only if $x \in V I(C, A)$ (see, e.g. [41]).

Let $C$ be a nonempty subset of $H$ and $k \in(0,1)$. A mapping $F: H \rightarrow H$ is called a $k$-contraction if $\|F(x)-F(y)\| \leq k\|x-y\|$ for all $x, y \in H$. It is easy to check that $P_{C}$ is a $k$-contraction on $C$. A subset $D$ of $H$ is called proximal if for each $x \in H$, there exists $y \in D$ such that

$$
\|x-y\|=d(x, D) .
$$

We denote by $C B(H), C C(H)$ and $P(H)$ the families of all nonempty closed bounded subsets of $H$, nonempty closed convex subset of $H$ and nonempty proximal bounded subsets of $H$ respectively. The Pompeiu-Hausdorff metric on $C B(H)$ is defined by

$$
H(A, B):=\max \left\{\sup _{x \in A} d(x, B), \sup _{y \in B} d(y, A)\right\}
$$


for all $A, B \in C B(H)$. Let $S: H \rightarrow 2^{H}$ be a multivalued mapping. An element $p \in H$ is called a fixed point of $S$ if $p \in S p$. We say that $S$ satisfies the endpoint condition if $S p=\{p\}$ for all $p \in F(S)$. For multivalued mappings $S_{i}: H \rightarrow 2^{H}(i \in \mathbb{N})$ with $\cap_{i=1}^{\infty} F\left(S_{i}\right) \neq \emptyset$, we say $S_{i}$ satisfies the common endpoint condition if $S_{i}(p)=\{p\}$ for all $i \in N, p \in \cap_{i=1}^{\infty} F\left(S_{i}\right)$. We recall some basic definitions of multivalued mappings.

Definition 2.2. A multivalued mapping $S: H \rightarrow C B(H)$ is said to be

1. nonexpansive if

$$
H(S x, S y) \leq\|x-y\|, \quad \text { for all } \quad x, y \in H,
$$

2. quasi-nonexpansive if $F(S) \neq \emptyset$ and

$$
H(S x, S p) \leq\|x-p\|, \quad \text { for all } \quad x \in H, p \in F(S),
$$

3. $\lambda$-demicontractive for $0 \leq \lambda<1$ if $F(S) \neq \emptyset$, and

$$
H(S x, S p)^{2} \leq\|x-p\|^{2}+\lambda d(x, S x)^{2}, \quad \text { for all } \quad x \in H, p \in F(S) .
$$

We note that the class of $\lambda$-demicontractive mappings includes several other types of classes of nonlinear mappings such as nonexpansive and quasi-nonexpansive. The best approximation operator $P_{S}$ for a multivalued mapping $S: H \rightarrow P(H)$ is defined by

$$
P_{S}(x):=\{y \in S x:\|x-y\|=d(x, S x)\} .
$$

One can easily prove that $F(S)=F\left(P_{S}\right)$ and $P_{S}$ satisfies the endpoint condition. However, Song and Cho [42] gave an example of a best approximation operator $P_{S}$ which is nonexpansive, but where $S$ is not necessarily nonexpansive.

Definition 2.3. Let $S: H \rightarrow C B(H)$ be a multivalued mapping. The multivalued mapping $I-S$ is said to be demiclosed at zero if for any sequence $\left\{x_{n}\right\} \subset H$ which converges weakly to $q$ and the sequence $\left\{\left\|x_{n}-u_{n}\right\|\right\}$ converges strongly to 0 , where $u_{n} \in S x_{n}$, then $q \in F(S)$.

The following results will be used in the sequel.

Lemma 2.4. [43, 44] In a real Hilbert space $H$, the following inequalities hold:

(i) $\|x-y\|^{2}=\|x\|^{2}-2\langle x, y\rangle+\|y\|^{2}$, for all $x, y \in H$,

(ii) $\|x+y\|^{2} \leq\|x\|^{2}+2\langle y, x+y\rangle$, for all $x, y \in H$.

Lemma 2.5. [45] Let $H$ be a real Hilbert space, $x_{i} \in H,(1 \leq i \leq m)$ and $\left\{\alpha_{i}\right\} \subset(0,1)$ with $\sum_{i=1}^{m} \alpha_{i}=1$. Then the following identity holds:

$$
\left\|\sum_{i=1}^{m} \alpha_{i} x_{i}\right\|=\sum_{i=1}^{m} \alpha_{i}\left\|x_{i}\right\|^{2}-\sum_{i, j=1, i \neq j}^{m} \alpha_{i} \alpha_{j}\left\|x_{i}-x_{j}\right\|^{2} .
$$

Lemma 2.6. [38] Let $\left\{\alpha_{n}\right\}$ and $\left\{\delta_{n}\right\}$ be sequences of nonnegative real numbers such that

$$
\alpha_{n+1} \leq\left(1-\delta_{n}\right) \alpha_{n}+\beta_{n}+\gamma_{n}, \quad n \geq 1,
$$

where $\left\{\delta_{n}\right\}$ is a sequence in $(0,1)$ and $\left\{\beta_{n}\right\}$ is a real sequence. Assume that $\sum_{n=0}^{\infty} \gamma_{n}<\infty$. Then, the following results hold:

(i) If $\beta_{n} \leq \delta_{n} M$ for some $M \geq 0$, then $\left\{\alpha_{n}\right\}$ is a bounded sequence.

(ii) If $\sum_{n=0}^{\infty} \delta_{n}=\infty$ and $\lim \sup _{n \rightarrow \infty} \frac{\beta_{n}}{\delta_{n}} \leq 0$, then $\lim _{n \rightarrow \infty} \alpha_{n}=0$. 
Lemma 2.7. [46] Let $\left\{a_{n}\right\}$ be a sequence of nonnegative real numbers satisfying the following relation:

$$
a_{n+1} \leq\left(1-\alpha_{n}\right) a_{n}+\alpha_{n} \sigma_{n}+\gamma_{n}, \quad n \geq 1,
$$

where

$$
\begin{aligned}
& \text { (i) }\left\{\alpha_{n}\right\} \subset[0,1] \text { and } \sum_{n=1}^{\infty} \alpha_{n}=\infty, \\
& \text { (ii) } \limsup _{n \rightarrow \infty} \sigma_{n} \leq 0, \\
& \text { (iii) } \gamma_{n} \geq 0,(n \geq 1) \text { and } \sum_{n=1}^{\infty} \gamma_{n}<\infty
\end{aligned}
$$

Then, $a_{n} \rightarrow 0$ as $n \rightarrow \infty$.

Lemma 2.8. [20] Let $\left\{a_{n}\right\}$ be a sequence of real numbers such that there exists a subsequence $\left\{n_{i}\right\}$ of $\{n\}$ with $a_{n_{i}}<a_{n_{i}+1}$ for all $i \in \mathbb{N}$. Consider the integer $\left\{m_{k}\right\}$ defined by

$$
m_{k}=\max \left\{j \leq k: a_{j}<a_{j+1}\right\} .
$$

Then $\left\{m_{k}\right\}$ is a nondecreasing sequence verifying $\lim _{n \rightarrow \infty} m_{n}=\infty$, and for all $k \in \mathbb{N}$, the following estimate holds:

$$
a_{m_{k}} \leq a_{m_{k}+1} \text {, and } a_{k} \leq a_{m_{k}+1} .
$$

\section{Main results}

In this section, we give a precise statement of our algorithm and discuss its strong convergence. Let $C$ be a nonempty, closed and convex subset of a real Hilbert space $H$ and $A: C \rightarrow H$ be a monotone and $L$-Lipschitz continuous mapping. For $i=1,2, \ldots, m$, let $S_{i}: H \rightarrow C B(H)$ be multivalued demicontractive mappings with constant $\kappa_{i}$ such that each $I-S_{i}$ are demiclosed at zero, $S_{i}(p)=\{p\}$ for all $p \in F\left(S_{i}\right)$, and $\kappa=\max \left\{\kappa_{i}\right\}$. Suppose

$$
\Gamma=V I(C, A) \cap \bigcap_{i=1}^{m} F\left(S_{i}\right) \neq \emptyset,
$$

and let $f: H \rightarrow H$ be a $\lambda$-contraction with constant $\lambda \in(0,1)$. Let $D$ be a bounded operator with coefficient $\rho>0$ such that $0<\xi<\frac{\rho}{\lambda}$ and let $\left\{\epsilon_{n}\right\},\left\{\beta_{n, i}\right\}$ and $\left\{\delta_{n}\right\}$ be nonnegative sequences such that $0<a \leq$ $\epsilon_{n}, \beta_{n, i}, \delta_{n} \leq b<1$, and let $\alpha \geq 3$.

\section{Algorithm 3.1.}

Step 0: Select initial guesses $x_{0}, x_{1} \in H$ and set $n=1$.

Step 1: Given the $(n-1)$ th and $n$th iterates, choose $\alpha_{n}$ such that we have $0 \leq \alpha_{n} \leq \tilde{\alpha}_{n}$ with $\tilde{\alpha}_{n}$ defined by

$$
\tilde{\alpha}_{n}=\left\{\begin{array}{l}
\min \left\{\frac{n-1}{n+\alpha-1}, \frac{\epsilon_{n}}{\left\|x_{n}-x_{n-1}\right\|}\right\}, \quad \text { if } x_{n} \neq x_{n-1}, \\
\frac{n-1}{n+\alpha-1}, \text { otherwise. }
\end{array}\right.
$$

Step 2: Compute

$$
\begin{aligned}
& u_{n}=x_{n}+\alpha_{n}\left(x_{n}-x_{n-1}\right), \\
& w_{n}=P_{C}\left(u_{n}-\mu_{n} A\left(u_{n}\right)\right),
\end{aligned}
$$

where $\mu_{n}=\bar{\sigma} \delta^{m_{n}}, \bar{\sigma}>0, \delta \in(0,1)$ and $m_{n}$ is the smallest nonnegative integer such that

$$
\left\|A\left(u_{n}\right)-A\left(w_{n}\right)\right\| \leq \frac{\eta\left\|u_{n}-w_{n}\right\|}{\mu_{n}}, \quad \eta \in(0,1) .
$$

Step 3: Construct the set $Q_{n}$ defined by

$$
Q_{n}=\left\{u \in H:\left\langle u_{n}-\mu_{n} A\left(u_{n}\right)-w_{n}, u-w_{n}\right\rangle \leq 0\right\},
$$


and compute

$$
\begin{cases}y_{n} & =P_{Q_{n}}\left(u_{n}-\mu_{n} A\left(w_{n}\right)\right), \\ z_{n} & =\beta_{n, 0} y_{n}+\sum_{i=1}^{m} \beta_{n, i} v_{n, i}, \\ x_{n+1} & =\delta_{n} \xi f\left(x_{n}\right)+\left(1-\delta_{n} D\right) z_{n},\end{cases}
$$

where $v_{n, i} \in S_{i} y_{n}$ and $\sum_{i=0}^{m} \beta_{n, i}=1$. Set $n:=n+1$ and go to Step 1 .

Remark 3.2. Observe that if $w_{n}=u_{n}=x_{n}$ and $x_{n} \in S_{i} x_{n}$, then we are at a common solution of the variational inequality (1) and a common fixed point of $S_{i}$, for all $i=1,2, \ldots, m$. In our convergence analysis, we will implicitly assume that this does not occur after finitely many iterations so that our Algorithm 3.1 generates an infinite sequence. We will see in the following result that the stepsize rule defined by (16) is well defined.

Lemma 3.3. [47] There exists a nonnegative integer $m_{n}$ satisfying (16). In addition

$$
\mu^{\star} \leq \mu_{n} \leq \bar{\sigma}, \quad \text { where } \mu^{\star}=\min \left\{\bar{\sigma}, \frac{\eta \delta}{L}\right\} .
$$

In order to establish our main result, we make the following assumption:

(C1) $\lim _{n \rightarrow \infty} \delta_{n}=0$ and $\sum_{n=0}^{\infty} \delta_{n}=\infty$,

(C2) $\quad \liminf _{n \rightarrow \infty}\left(\beta_{n, 0}-\kappa\right) \beta_{n, i}>0$ for all $i=1,2, \ldots, m$,

(C3) $\epsilon_{n}=o\left(\delta_{n}\right)$, i.e., $\lim _{n \rightarrow \infty} \frac{\epsilon_{n}}{\delta_{n}}=0\left(\right.$ e.g. $\left.\epsilon_{n}=\frac{1}{(n+1)^{2}}, \delta_{n}=\frac{1}{n+1}\right)$.

Remark 3.4. Observe that from (14) and Assumptions (C3), we have

$$
\lim _{n \rightarrow \infty} \alpha_{n}\left\|x_{n}-x_{n-1}\right\|=0 \text { and } \lim _{n \rightarrow \infty} \frac{\alpha_{n}}{\delta_{n}}\left\|x_{n}-x_{n-1}\right\|=0
$$

Also, note that Step 1 in our Algorithm 3.1 is easily implemented in numerical computation since the value of $\left\|x_{n}-x_{n-1}\right\|$ is known a prori before choosing $\alpha_{n}$.

We proceed to prove the following lemmas before proving the convergence of our main Algorithm 3.1.

Lemma 3.5. The sequence $\left\{x_{n}\right\}$ generated by Algorithm 3.1 is bounded.

Proof. Let $p \in \Gamma$, then

$$
\begin{aligned}
\left\|u_{n}-p\right\| & =\left\|x_{n}+\alpha_{n}\left(x_{n}-x_{n-1}\right)-p\right\| \\
& \leq\left\|x_{n}-p\right\|+\alpha_{n}\left\|x_{n}-x_{n-1}\right\| .
\end{aligned}
$$

Also from (11) and (15), we get

$$
\begin{aligned}
\left\|y_{n}-p\right\|^{2}= & \left\|P_{Q_{n}}\left(u_{n}-\mu_{n} A w_{n}\right)-p\right\|^{2} \\
\leq & \left\|u_{n}-\mu_{n} A w_{n}-p\right\|^{2}-\left\|u_{n}-\mu_{n} A w_{n}-y_{n}\right\|^{2} \\
= & \left\|u_{n}-\mu_{n} A w_{n}\right\|^{2}-2\left\langle u_{n}-\mu_{n} A w_{n}, p\right\rangle+\|p\|^{2} \\
& -\left[\left\|u_{n}-\mu_{n} A w_{n}\right\|^{2}-2\left\langle u_{n}-\mu_{n} A w_{n}, y_{n}\right\rangle+\left\|y_{n}\right\|^{2}\right] \\
= & \|p\|^{2}-2\left\langle u_{n}, p\right\rangle+\left\|u_{n}\right\|^{2}-\left\|u_{n}\right\|^{2}+2 \mu_{n}\left\langle A w_{n}, p\right\rangle \\
& +2\left\langle u_{n}, y_{n}\right\rangle-2 \mu_{n}\left\langle A w_{n}, y_{n}\right\rangle-\left\|y_{n}\right\|^{2} \\
= & \left\|u_{n}-p\right\|^{2}-\left\|u_{n}-y_{n}\right\|^{2}+2 \mu_{n}\left\langle A w_{n}, p-y_{n}\right\rangle .
\end{aligned}
$$

Since $A$ is monotone, then

$$
\left\langle A w_{n}-A p, w_{n}-p\right\rangle \geq 0, \quad \text { for all } n \geq 1 \text {, }
$$


and hence

$$
\left\langle A w_{n}, w_{n}-p\right\rangle \geq\left\langle A p, w_{n}-p\right\rangle .
$$

This implies that

$$
\left\langle A w_{n}, w_{n}-p\right\rangle \geq\left\langle A p, w_{n}-p\right\rangle \geq 0 .
$$

Therefore, we have

$$
\begin{aligned}
0 & \leq\left\langle A w_{n}, w_{n}-p\right\rangle \\
& =\left\langle A w_{n}, w_{n}-y_{n}+y_{n}-p\right\rangle \\
& =\left\langle A w_{n}, w_{n}-y_{n}\right\rangle+\left\langle A w_{n}, y_{n}-p\right\rangle
\end{aligned}
$$

Whence

$$
\left\langle A w_{n}, p-y_{n}\right\rangle \leq\left\langle A w_{n}, w_{n}-y_{n}\right\rangle .
$$

Substituting (21) into (20), we get

$$
\begin{aligned}
\left\|y_{n}-p\right\|^{2} & \leq\left\|u_{n}-p\right\|^{2}-\left\|u_{n}-y_{n}\right\|^{2}+2 \mu_{n}\left\langle A w_{n}, w_{n}-y_{n}\right\rangle \\
& =\left\|u_{n}-p\right\|^{2}-\left\|u_{n}-w_{n}+w_{n}-y_{n}\right\|^{2}+2 \mu_{n}\left\langle A w_{n}, w_{n}-y_{n}\right\rangle \\
& =\left\|u_{n}-p\right\|^{2}-\left[\left\|u_{n}-w_{n}\right\|^{2}+2\left\langle u_{n}-w_{n}, w_{n}-y_{n}\right\rangle+\left\|w_{n}-y_{n}\right\|^{2}\right]+2 \mu_{n}\left\langle A w_{n}, w_{n}-y_{n}\right\rangle \\
& =\left\|u_{n}-p\right\|^{2}-\left\|u_{n}-w_{n}\right\|^{2}-\left\|w_{n}-y_{n}\right\|^{2}+2\left\langle u_{n}-\mu_{n} A w_{n}-w_{n}, y_{n}-w_{n}\right\rangle .
\end{aligned}
$$

Using (16), we get

$$
\begin{aligned}
\left\langle u_{n}-\mu_{n} A w_{n}-w_{n}, y_{n}-w_{n}\right\rangle= & \left\langle u_{n}-\mu_{n} A u_{n}-w_{n}, y_{n}-w_{n}\right\rangle \\
& +\left\langle\mu_{n} A u_{n}-\mu_{n} A w_{n}, y_{n}-w_{n}\right\rangle \\
\leq & \mu_{n}\left\langle A u_{n}-A w_{n}, y_{n}-w_{n}\right\rangle \\
\leq & \eta\left\|u_{n}-w_{n}\right\| \cdot\left\|y_{n}-w_{n}\right\| .
\end{aligned}
$$

Hence from (22) and (23), we get

$$
\begin{aligned}
\left\|y_{n}-p\right\|^{2} & \leq\left\|u_{n}-p\right\|^{2}-\left\|u_{n}-w_{n}\right\|^{2}-\left\|w_{n}-y_{n}\right\|^{2}+2 \eta\left\|u_{n}-w_{n}\right\| \cdot\left\|y_{n}-w_{n}\right\| \\
& \leq\left\|u_{n}-p\right\|^{2}-\left\|u_{n}-w_{n}\right\|^{2}-\left\|w_{n}-y_{n}\right\|^{2}+\eta\left(\left\|u_{n}-w_{n}\right\|^{2}+\left\|w_{n}-y_{n}\right\|^{2}\right) \\
& =\left\|u_{n}-p\right\|^{2}-(1-\eta)\left\|u_{n}-w_{n}\right\|^{2}-(1-\eta)\left\|w_{n}-y_{n}\right\|^{2} .
\end{aligned}
$$

Thus

$$
\left\|y_{n}-p\right\|^{2} \leq\left\|u_{n}-p\right\|^{2}
$$

Furthermore, using Lemma 2.5, we have

$$
\begin{aligned}
\left\|z_{n}-p\right\|^{2} & =\left\|\beta_{n, 0} y_{n}+\sum_{i=1}^{m} \beta_{n, i} v_{n, i}-p\right\|^{2} \\
& \leq \beta_{n, 0}\left\|y_{n}-p\right\|^{2}+\sum_{i=1}^{m} \beta_{n, i}\left\|v_{n, i}-p\right\|^{2}-\sum_{i=1}^{m} \beta_{n, 0} \beta_{n, i}\left\|y_{n}-v_{n, i}\right\|^{2} \\
& =\beta_{n, 0}\left\|y_{n}-p\right\|^{2}+\sum_{i=1}^{m} \beta_{n, i} d\left(v_{n, i}, S_{i} p\right)^{2}-\sum_{i=1}^{m} \beta_{n, 0} \beta_{n, i}\left\|y_{n}-v_{n, i}\right\|^{2} \\
& \leq \beta_{n, 0}\left\|y_{n}-p\right\|^{2}+\sum_{i=1}^{m} \beta_{n, i} H\left(S_{i} y_{n}, S_{i} p\right)^{2}-\sum_{i=1}^{m} \beta_{n, 0} \beta_{n, i}\left\|y_{n}-v_{n, i}\right\|^{2} \\
& \leq \beta_{n, 0}\left\|y_{n}-p\right\|^{2}+\sum_{i=1}^{m} \beta_{n, i}\left(\left\|y_{n}-p\right\|^{2}+\kappa_{i} d\left(y_{n}, S_{i} y_{n}\right)^{2}\right)-\sum_{i=1}^{m} \beta_{n, 0} \beta_{n, i}\left\|y_{n}-v_{n, i}\right\|^{2}
\end{aligned}
$$




$$
\begin{aligned}
& \leq \beta_{n, 0}\left\|y_{n}-p\right\|^{2}+\sum_{i=1}^{m} \beta_{n, i}\left\|y_{n}-p\right\|^{2}+\sum_{i=1}^{m} \beta_{n, i} \kappa \mid\left\|y_{n}-v_{n, i}\right\|^{2}-\sum_{i=1}^{m} \beta_{n, 0} \beta_{n, i}\left\|y_{n}-v_{n, i}\right\|^{2} \\
& =\left\|y_{n}-p\right\|^{2}-\sum_{i=1}^{m}\left(\beta_{n, 0}-\kappa\right) \beta_{n, i}\left\|y_{n}-v_{n, i}\right\|^{2},
\end{aligned}
$$

and by condition (C2), we get

$$
\left\|z_{n}-p\right\|^{2} \leq\left\|y_{n}-p\right\|^{2} .
$$

Therefore, from (19), (25) and (27), we have

$$
\begin{aligned}
\left\|x_{n+1}-p\right\|= & \left\|\delta_{n}\left(\xi f\left(x_{n}\right)-D p\right)+\left(1-\delta_{n} D\right)\left(z_{n}-p\right)\right\| \\
\leq & \delta_{n}\left\|\xi f\left(x_{n}\right)-D p\right\|+\left(1-\delta_{n} \rho\right)\left\|z_{n}-p\right\| \\
\leq & \delta_{n}\left[\left\|\xi\left(f\left(x_{n}\right)-f(p)\right)+(\xi f(p)-D p)\right\|\right]+\left(1-\delta_{n} \rho\right)\left\|z_{n}-p\right\| \\
\leq & \delta_{n} \xi \lambda\left\|x_{n}-p\right\|+\delta_{n}\|\xi f(p)-D p\|+\left(1-\delta_{n} \rho\right)\left[\left\|x_{n}-p\right\|+\alpha_{n}\left\|x_{n}-x_{n-1}\right\|\right] \\
= & \left(1-\delta_{n}(\rho-\xi \lambda)\right)\left\|x_{n}-p\right\|+\delta_{n}\|\xi f(p)-D p\|+\left(1-\delta_{n} \rho\right) \alpha_{n}\left\|x_{n}-x_{n-1}\right\| \\
= & \left(1-\delta_{n}(\rho-\xi \lambda)\right)\left\|x_{n}-p\right\|+(\rho-\xi \lambda) \delta_{n}\left\{\frac{\|\xi f(p)-D p\|}{\rho-\xi \lambda}\right. \\
& \left.+\left(\frac{1-\delta_{n} \rho}{\rho-\xi \lambda}\right) \frac{\alpha_{n}}{\delta_{n}}\left\|x_{n}-x_{n-1}\right\|\right\} .
\end{aligned}
$$

Note that $\sup _{n \geq 1}\left(\frac{1-\delta_{n} \rho}{\rho-\xi \lambda}\right) \frac{\alpha_{n}}{\delta_{n}}\left\|x_{n}-x_{n-1}\right\|$ exists by Remark 3.4 and let

$$
M:=\max \left\{\frac{\|\xi f(p)-D p\|}{\|\rho-\xi \lambda\|}, \sup _{n \geq 1}\left(\frac{1-\delta_{n} \rho}{\rho-\xi \lambda}\right) \frac{\alpha_{n}}{\delta_{n}}\left\|x_{n}-x_{n-1}\right\|\right\} .
$$

Then we have

$$
\left\|x_{n+1}-p\right\| \leq\left(1-\delta_{n}(\rho-\xi \lambda)\right)\left\|x_{n}-p\right\|+\delta_{n}(\rho-\xi \lambda) M .
$$

Using Lemma 2.6(i) and (29), we have $\left\{\left\|x_{n}-p\right\|\right\}$ is bounded and thus $\left\{x_{n}\right\}$ is bounded. Consequently, $\left\{u_{n}\right\},\{A u n\},\left\{w_{n}\right\},\left\{y_{n}\right\}$ and $\left\{z_{n}\right\}$ are all bounded.

Lemma 3.6. Let $\left\{x_{n}\right\}$ be a sequence generated by Algorithm 3.1. Put $s_{n}=\left\|x_{n}-p\right\|^{2}, \quad \tilde{a}_{n}=\frac{2 \delta_{n}(\rho-\xi \lambda)}{1-\delta_{n} \xi \lambda}$, $b_{n}=\frac{1}{2(\rho-\xi \lambda)}\left(2\left\langle\xi f(p)-D p, x_{n+1}-p\right\rangle+\delta_{n} M_{1}\right)$, for some $M_{1}>0$ and $c_{n}=\frac{\alpha_{n}\left\|x_{n}-x_{n-1}\right\|}{1-\delta_{n} \xi \lambda} M_{2}$, where $M_{2}=\sup _{n \geq 1}\left(\left(1-\delta_{n} \rho\right)^{2}\left(\left\|x_{n}-p\right\|+\left\|x_{n-1}-p\right\|\right)+2\left(1-\delta_{n} \rho\right)^{2}|| x_{n}-x_{n-1} \|\right)$ and $p \in \Gamma$. Then, the following estimates hold:

(i) $s_{n+1} \leq\left(1-\tilde{a}_{n}\right) s_{n}+\tilde{a}_{n} b_{n}+c_{n}$,

(ii) $-1 \leq \lim \sup _{n \rightarrow \infty} b_{n}<+\infty$,

Proof. From (15), we have

$$
\begin{aligned}
\left\|u_{n}-p\right\|^{2} & =\left\|x_{n}+\alpha_{n}\left(x_{n}-x_{n-1}\right)-p\right\|^{2} \\
& =\left\|x_{n}-p\right\|^{2}+2 \alpha_{n}\left\langle x_{n}-p, x_{n}-x_{n-1}\right\rangle+\alpha_{n}^{2}\left\|x_{n}-x_{n-1}\right\|^{2} .
\end{aligned}
$$

Using Lemma 2.4(i), we have

$$
2\left\langle x_{n}-p, x_{n}-x_{n-1}\right\rangle=-\left\|x_{n-1}-p\right\|^{2}+\left\|x_{n}-p\right\|^{2}+\left\|x_{n}-x_{n-1}\right\|^{2},
$$

thus, substituting (31) into (30), we get

$$
\left\|u_{n}-p\right\|^{2}=\left\|x_{n}-p\right\|^{2}+\alpha_{n}\left(-\left\|x_{n-1}-p\right\|^{2}+\left\|x_{n}-p\right\|^{2}+\left\|x_{n}-x_{n-1}\right\|^{2}\right)+\alpha_{n}^{2}\left\|x_{n}-x_{n-1}\right\|^{2}
$$




$$
\leq\left\|x_{n}-p\right\|^{2}+\alpha_{n}\left(\left\|x_{n}-p\right\|^{2}-\left\|x_{n-1}-p\right\|^{2}\right)+2 \alpha_{n}\left\|x_{n}-x_{n-1}\right\|^{2} \text {. }
$$

Now, from Lemma 2.4(ii), we have

$$
\begin{aligned}
\left\|x_{n+1}-p\right\|^{2} & =\left\|\delta_{n}\left(\xi f\left(x_{n}\right)-D p\right)+\left(1-\delta_{n} D\right)\left(z_{n}-p\right)\right\|^{2} \\
& \leq\left(1-\delta_{n} \rho\right)^{2}\left\|z_{n}-p\right\|^{2}+2 \delta_{n}\left\langle\xi f\left(x_{n}\right)-D p, x_{n+1}-p\right\rangle
\end{aligned}
$$

It follows from (25), (27) and (30) that

$$
\begin{aligned}
\left\|x_{n+1}-p\right\|^{2} \leq & \left(1-\delta_{n} \rho\right)^{2}\left\|u_{n}-p\right\|^{2}+2 \delta_{n}\left\langle\xi f\left(x_{n}\right)-D p, x_{n+1}-p\right\rangle \\
= & \left(1-\delta_{n} \rho\right)^{2}\left(\left\|x_{n}-p\right\|^{2}+\alpha_{n}\left(\left\|x_{n}-p\right\|^{2}-\left\|x_{n-1}-p\right\|^{2}\right)+2 \alpha_{n}\left\|x_{n}-x_{n-1}\right\|^{2}\right) \\
& +2 \delta_{n}\left\langle\xi f\left(x_{n}\right)-D p, x_{n+1}-p\right\rangle \\
= & \left(1-\delta_{n} \rho\right)^{2}\left\|x_{n}-p\right\|^{2}+\alpha_{n}\left(1-\delta_{n} \rho\right)^{2}\left(\left\|x_{n}-p\right\|^{2}-\left\|x_{n-1}-p\right\|^{2}\right)+2 \alpha_{n}\left(1-\delta_{n} \rho\right)^{2}\left\|x_{n}-x_{n-1}\right\|^{2} \\
& +2 \delta_{n}\left\langle\xi f\left(x_{n}\right)-D p, x_{n+1}-p\right\rangle \\
\leq & \left(1-\delta_{n} \rho\right)^{2}\left\|x_{n}-p\right\|^{2}+\alpha_{n}\left(1-\delta_{n} \rho\right)^{2}\left(\left\|x_{n}-p\right\|+\left\|x_{n-1}-p\right\|\right)\left\|x_{n}-x_{n-1}\right\| \\
& +2 \alpha_{n}\left(1-\delta_{n} \rho\right)^{2}\left\|x_{n}-x_{n-1}\right\|^{2}+2 \delta_{n}\left\langle\xi f\left(x_{n}\right)-D p, x_{n+1}-p\right\rangle .
\end{aligned}
$$

Also

$$
\begin{aligned}
2\left\langle\xi f\left(x_{n}\right)-D p, x_{n+1}-p\right\rangle & =2\left\langle\xi\left(f\left(x_{n}\right)-f(p)\right)+\xi f(p)-D p, x_{n+1}-p\right\rangle \\
& \leq 2 \xi \lambda\left\|x_{n}-p\right\| \cdot\left\|x_{n+1}-p\right\|+2\left\langle\xi f(p)-D p, x_{n+1}-p\right\rangle \\
& \leq \xi \lambda\left(\left\|x_{n}-p\right\|^{2}+\left\|x_{n+1}-p\right\|^{2}\right)+2\left\langle\xi f(p)-D p, x_{n+1}-p\right\rangle .
\end{aligned}
$$

Substituting (35) into (34), we have

$$
\begin{aligned}
\left\|x_{n+1}-p\right\|^{2} \leq & {\left[\left(1-\delta_{n} \rho\right)^{2}+\delta_{n} \xi \lambda\right]\left\|x_{n}-p\right\|^{2}+\alpha_{n}\left(1-\delta_{n} \rho\right)^{2}\left(\left\|x_{n}-p\right\|+\left\|x_{n-1}-p\right\|\right)\left\|x_{n}-x_{n-1}\right\| } \\
& +2 \alpha_{n}\left(1-\delta_{n} \rho\right)^{2}\left\|x_{n}-x_{n-1}\right\|^{2}+\delta_{n} \xi \lambda\left\|x_{n+1}-p\right\|^{2}+2 \delta_{n}\left\langle\xi f(p)-D p, x_{n+1}-p\right\rangle \\
= & \left(1-\delta_{n}(2 \rho-\xi \lambda)\right)\left\|x_{n}-p\right\|^{2}+\left(\delta_{n} \rho\right)^{2}\left\|x_{n}-p\right\|^{2}+\alpha_{n}\left[\left(1-\delta_{n} \rho\right)^{2}\left(\left\|x_{n}-p\right\|+\left\|x_{n-1}-p\right\|\right)\right. \\
& \left.+2\left(1-\delta_{n} \rho\right)^{2}\left\|x_{n}-x_{n-1}\right\|\right]\left\|x_{n}-x_{n-1}\right\|+\delta_{n} \xi \lambda\left\|x_{n+1}-p\right\|^{2}+2 \delta_{n}\left\langle\xi f(p)-D p, x_{n+1}-p\right\rangle \\
\leq & \left(1-\delta_{n}(2 \rho-\xi \lambda)\right)\left\|x_{n}-p\right\|^{2}+\delta_{n} \xi \lambda\left\|x_{n+1}-p\right\|^{2}+\alpha_{n}\left[\left(1-\delta_{n} \rho\right)^{2}\left(\left\|x_{n}-p\right\|+\left\|x_{n-1}-p\right\|\right)\right. \\
& \left.+2\left(1-\delta_{n} \rho\right)^{2}\left\|x_{n}-x_{n-1}\right\|\right]\left\|x_{n}-x_{n-1}\right\|+\delta_{n}\left(2\left\langle\xi f(p)-D p, x_{n+1}-p\right\rangle+\delta_{n} M_{1}\right)
\end{aligned}
$$

for some $M_{1} \geq 0$. Hence

$$
\begin{aligned}
\left\|x_{n+1}-p\right\|^{2} \leq & \frac{\left(1-\delta_{n}(2 \rho-\xi \lambda)\right)}{1-\delta_{n} \xi \lambda}\left\|x_{n}-p\right\|^{2}+\frac{\alpha_{n}}{1-\delta_{n} \xi \lambda}\left\|x_{n}-x_{n-1}\right\| M_{2}+\frac{\delta_{n}\left(2\left\langle\xi f(p)-D p, x_{n+1}-p\right\rangle+\delta_{n} M_{1}\right)}{1-\delta_{n} \xi \lambda} \\
= & \left(1-\frac{2 \delta_{n}(\rho-\xi \lambda)}{1-\delta_{n} \xi \lambda}\right)\left\|x_{n}-p\right\|^{2}+\frac{\alpha_{n}}{1-\delta_{n} \xi \lambda}\left\|x_{n}-x_{n-1}\right\| M_{2} \\
& +\frac{2 \delta_{n}(\rho-\xi \lambda)}{1-\delta_{n} \xi \lambda} \times \frac{\left(2\left\langle\xi f(p)-D p, x_{n+1}-p\right\rangle+\delta_{n} M_{1}\right)}{2(\rho-\xi \lambda)} .
\end{aligned}
$$

This establishes (i). Next, we prove (ii). Since $\left\{x_{n}\right\}$ is bounded and $\delta_{n} \in(0,1)$, then we have

$$
\sup _{n \geq 0} b_{n} \leq \sup _{n \geq 0} \frac{1}{2(\rho-\xi \lambda)}\left(2\left\|\xi f(p)-D x^{\star}\right\| \cdot\left\|x_{n+1}-p\right\|+M_{1}\right)<\infty .
$$

We next show that $\limsup _{n \rightarrow \infty} b_{n} \geq-1$. Assume the contrary, i.e suppose $\lim \sup _{n \rightarrow \infty} b_{n}<-1$, which implies that there exists $n_{0} \in \mathbb{N}$ such that $b_{n} \leq-1$ for all $n \geq n_{0}$. Hence, it follows from (i) that

$$
s_{n+1} \leq\left(1-\tilde{a}_{n}\right) s_{n}+\tilde{a}_{n} b_{n}+c_{n}
$$




$$
\begin{aligned}
& <\left(1-\tilde{a}_{n}\right) s_{n}-\tilde{a}_{n}+c_{n} \\
& =s_{n}-\tilde{a}_{n}\left(s_{n}+1\right)+c_{n} \\
& \leq s_{n}-2(\rho-\xi \lambda) \delta_{n}+c_{n} .
\end{aligned}
$$

By induction, we get

$$
s_{n+1} \leq s_{n_{0}}-2(\rho-\xi \lambda) \sum_{i=n_{0}}^{n} \delta_{i}+c_{n} \quad \text { for all } n \geq n_{0} .
$$

Taking lim sup of both sides in the last inequality (noting that $c_{n} \rightarrow 0$ ), we have

$$
\limsup _{n \rightarrow \infty} s_{n} \leq s_{n_{0}}-\lim _{n \rightarrow \infty} 2(\rho-\xi \lambda) \sum_{i=n_{0}}^{n} \delta_{i}=-\infty .
$$

This contradicts the fact that $\left\{s_{n}\right\}$ is a nonnegative real sequence. Therefore, $\lim _{\sup _{n \rightarrow \infty}} b_{n} \geq-1$.

Remark 3.7. Since $\delta_{n} \rightarrow 0$ as $n \rightarrow \infty$, it is easy to check that $\tilde{a}_{n} \rightarrow 0$, and by Remark 3.4, $c_{n} \rightarrow 0$ as $n \rightarrow \infty$.

We next state and prove our main theorem.

Theorem 3.8. Let $C$ be a nonempty, closed and convex subset of a real Hilbert space $H$, and let $A: C \rightarrow H$ be a monotone and L-Lipschitz continuous mapping. For each $i=1,2, \ldots, m$, let $S_{i}: H \rightarrow C B(H)$ be multivalued demicontractive mappings with constant $\kappa_{i}$ such that each $I-S_{i}$ are demiclosed at zero, $S_{i}(p)=\{p\}$ for all $p \in F\left(S_{i}\right), i=1,2, \ldots, m$ and $\kappa=\max \left\{\kappa_{i}\right\}$. Suppose $\Gamma=V I(C, A) \cap \bigcap_{i=1}^{m} F\left(S_{i}\right) \neq \emptyset$. Let $f: H \rightarrow H$ be a $\lambda$ contraction with constant $\lambda \in(0,1)$ and $D$ be a bounded operator with coefficient $\rho>0$ such that $0<\xi<\frac{\rho}{\lambda}$. Let $\left\{x_{n}\right\}$ be generated by Algorithm 3.1 and Assumptions (C1)-(C3) are satisfied. Then the sequence $\left\{x_{n}\right\}$ converges strongly to a point $z$, where $z=P_{\Gamma}(I-D+\xi f)(z)$ is a unique solution of the variational inequality

$$
\langle(D-\xi f) z, z-x\rangle \leq 0, \quad x \in \Gamma
$$

Proof. Let $p \in \Gamma$ and denote $\left\|x_{n}-p\right\|^{2}$ by $\Phi_{n}$. We consider the following two possible cases.

CASE A: Suppose there exists $n_{0} \in \mathbb{N}$ such that $\Phi_{n}$ is monotonically nonincreasing for all $n \geq n_{0}$. Since $\Phi_{n}$ is bounded, then it is convergent and so $\Phi_{n}-\Phi_{n+1} \rightarrow 0$ as $n \rightarrow \infty$. We first show that $\left\|w_{n}-u_{n}\right\| \rightarrow 0$, $\left\|v_{n, i}-y_{n}\right\| \rightarrow 0$ and $\left\|x_{n+1}-x_{n}\right\| \rightarrow 0$, as $n \rightarrow \infty$. From (24), (27) and (32), we have

$$
\begin{aligned}
\left\|x_{n+1}-p\right\|^{2} \leq & \left(1-\delta_{n} \rho\right)^{2}\left\|z_{n}-p\right\|^{2}+2 \delta_{n}\left\langle\xi f\left(x_{n}\right)-D p, x_{n+1}-p\right\rangle \\
\leq & \left(1-\delta_{n} \rho\right)^{2}\left\{\left\|u_{n}-p\right\|^{2}-(1-\eta)\left\|u_{n}-w_{n}\right\|^{2}-(1-\eta)\left\|w_{n}-y_{n}\right\|^{2}\right\} \\
& +2 \delta_{n}\left\langle\xi f\left(x_{n}\right)-D p, x_{n+1}-p\right\rangle \\
\leq & \left(1-\delta_{n} \rho\right)^{2}\left\{\left\|x_{n}-p\right\|^{2}+\alpha_{n}\left(\left\|x_{n}-p\right\|^{2}-\left\|x_{n-1}-p\right\|^{2}\right)+2 \alpha_{n}\left\|x_{n}-x_{n-1}\right\|^{2}\right. \\
& \left.-(1-\eta)\left\|u_{n}-w_{n}\right\|^{2}-(1-\eta)\left\|w_{n}-y_{n}\right\|^{2}\right\} \\
& +2 \delta_{n}\left\langle\xi f\left(x_{n}\right)-D p, x_{n+1}-p\right\rangle .
\end{aligned}
$$

Therefore

$$
\begin{aligned}
\left(1-\delta_{n} \rho\right)^{2}(1-\eta) & \left\|u_{n}-w_{n}\right\|^{2} \leq\left(1-\delta_{n} \rho\right)^{2}\left\|x_{n}-p\right\|^{2}+\alpha_{n}\left(1-\delta_{n} \rho\right)^{2}\left(\left\|x_{n}-p\right\|^{2}-\left\|x_{n-1}-p\right\|^{2}\right) \\
& +2 \alpha_{n}\left(1-\delta_{n} \rho\right)^{2}\left\|x_{n}-x_{n-1}\right\|^{2}+2 \delta_{n}\left\langle\xi f\left(x_{n}\right)-D p, x_{n+1}-p\right\rangle-\left\|x_{n+1}-p\right\|^{2} \\
& \leq \Phi_{n}-\Phi_{n+1}+\delta_{n} M_{3}+\alpha_{n}\left(1-\delta_{n} \rho\right)^{2}\left(\Phi_{n}-\Phi_{n-1}\right) \\
& +2 \alpha_{n}\left(1-\delta_{n} \rho\right)^{2}\left\|x_{n}-x_{n-1}\right\|+2 \delta_{n}\left\langle\xi f\left(x_{n}\right)-D p, x_{n+1}-p\right\rangle \rightarrow 0,
\end{aligned}
$$

as $n \rightarrow \infty$ for some $M_{3}>0$. Since $\delta_{n} \rightarrow 0$ as $n \rightarrow \infty$ and $\eta \in(0,1)$, then

$$
\lim _{n \rightarrow \infty}\left\|u_{n}-w_{n}\right\|=0
$$


Similarly, from (37), we can also show that

$$
\lim _{n \rightarrow \infty}\left\|w_{n}-y_{n}\right\|=0 .
$$

Clearly from (15), we get

$$
\begin{aligned}
\left\|u_{n}-x_{n}\right\| & =\left\|x_{n}+\alpha_{n}\left(x_{n}-x_{n-1}\right)-x_{n}\right\| \\
& =\alpha_{n}\left\|x_{n}-x_{n-1}\right\| \rightarrow 0, \quad n \rightarrow \infty
\end{aligned}
$$

Therefore

$$
\lim _{n \rightarrow \infty}\left\|w_{n}-x_{n}\right\| \leq \lim _{n \rightarrow \infty}\left(\left\|w_{n}-u_{n}\right\|+\left\|u_{n}-x_{n}\right\|\right)=0
$$

and

$$
\lim _{n \rightarrow \infty}\left\|y_{n}-x_{n}\right\| \leq \lim _{n \rightarrow \infty}\left(\left\|y_{n}-w_{n}\right\|+\left\|w_{n}-x_{n}\right\|\right)=0 .
$$

Also from (25), (26), (32) and (33), we obtain

$$
\begin{aligned}
\left\|x_{n+1}-p\right\|^{2} \leq & \left(1-\delta_{n} \rho\right)^{2}\left\|z_{n}-p\right\|^{2}+2 \delta_{n}\left\langle\xi f\left(x_{n}\right)-D p, x_{n+1}-p\right\rangle \\
\leq & \left(1-\delta_{n} \rho\right)^{2}\left\{\left\|y_{n}-p\right\|^{2}-\sum_{i=1}^{m}\left(\beta_{n, 0}-\kappa\right) \beta_{n, i}\left\|y_{n}-v_{n, i}\right\|^{2}\right\} \\
& +2 \delta_{n}\left\langle\xi f\left(x_{n}\right)-D p, x_{n+1}-p\right\rangle \\
\leq & \left(1-\delta_{n} \rho\right)^{2}\left\{\left\|x_{n}-p\right\|^{2}+\alpha_{n}\left(\left\|x_{n}-p\right\|^{2}-\left\|x_{n-1}-p\right\|^{2}\right)+2 \alpha_{n}\left\|x_{n}-x_{n-1}\right\|^{2}\right. \\
& \left.-\sum_{i=1}^{m}\left(\beta_{n, 0}-\kappa\right) \beta_{n, i}\left\|y_{n}-v_{n, i}\right\|^{2}\right\}+2 \delta_{n}\left\langle\xi f\left(x_{n}\right)-D p, x_{n+1}-p\right\rangle .
\end{aligned}
$$

Hence

$$
\begin{aligned}
\left(1-\delta_{n} \rho\right)^{2} & \sum_{i=1}^{m}\left(\beta_{n, 0}-\kappa\right) \beta_{n, i}\left\|y_{n}-v_{n, i}\right\|^{2} \leq\left(1-\delta_{n} \rho\right)^{2}\left\|x_{n}-p\right\|^{2}+\alpha_{n}\left(1-\delta_{n} \rho\right)^{2}\left(\left\|x_{n}-p\right\|^{2}-\left\|x_{n-1}-p\right\|^{2}\right) \\
& +2 \alpha_{n}\left(1-\delta_{n} \rho\right)^{2}\left\|x_{n}-x_{n-1}\right\|^{2}++2 \delta_{n}\left\langle\xi f\left(x_{n}\right)-D p, x_{n+1}-p\right\rangle-\left\|x_{n+1}-p\right\|^{2} \\
& \leq \Phi_{n}-\Phi_{n+1}+\delta_{n} M_{3}+\alpha_{n}\left(1-\delta_{n} \rho\right)^{2}\left(\Phi_{n}-\Phi_{n-1}\right)+2 \alpha_{n}\left(1-\delta_{n} \rho\right)^{2}\left\|x_{n}-x_{n-1}\right\|^{2} \\
& +2 \delta_{n}\left\langle\xi f\left(x_{n}\right)-D p, x_{n+1}-p\right\rangle \rightarrow 0, \quad \text { as } n \rightarrow \infty .
\end{aligned}
$$

Therefore, using condition (C2), it follows that

$$
\lim _{n \rightarrow \infty}\left\|y_{n}-v_{n, i}\right\|=0
$$

Also

$$
\begin{aligned}
\left\|z_{n}-y_{n}\right\| & =\left\|\beta_{n, 0} y_{n}+\sum_{i=1}^{m} \beta_{n, i} v_{n, i}-y_{n}\right\| \\
& \leq \beta_{n, 0}\left\|y_{n}-y_{n}\right\|+\sum_{i=1}^{m} \beta_{m, i}\left\|v_{n, i}-y_{n}\right\| \rightarrow 0,
\end{aligned}
$$

therefore

$$
\lim _{n \rightarrow \infty}|| z_{n}-x_{n} \|=\lim _{n \rightarrow \infty}\left(\left\|z_{n}-y_{n}\right\|+\left\|y_{n}-x_{n}\right\|\right)=0 .
$$

Now from (15) and condition (C1), we get

$$
\begin{aligned}
\left\|x_{n+1}-z_{n}\right\| & =\left\|\delta_{n} \xi f\left(x_{n}\right)+\left(1-\delta_{n} D\right) z_{n}-z_{n}\right\| \\
& =\delta_{n}\left\|\xi f\left(x_{n}\right)-D z_{n}\right\| \rightarrow 0,
\end{aligned}
$$


and therefore

$$
\left\|x_{n+1}-x_{n}\right\| \leq\left\|x_{n+1}-z_{n}\right\|+\left\|z_{n}-x_{n}\right\| \rightarrow 0, \quad n \rightarrow \infty .
$$

Next, we show that $\Omega_{w}\left(x_{n}\right) \subset V I(C, A) \cap \bigcap_{i=1}^{m} F\left(S_{i}\right)$, where $\Omega_{w}\left(x_{n}\right)$ is the weak subsequential limit of $\left\{x_{n}\right\}$. Let $\bar{x} \in \Omega_{w}\left(x_{n}\right)$, and observe that there exists a subsequence $\left\{x_{n_{j}}\right\}$ of $\left\{x_{n}\right\}$ such that $x_{n_{j}} \rightarrow \bar{x}$ as $j \rightarrow \infty$. Let $\left\{w_{n_{j}}\right\}$ and $\left\{u_{n_{j}}\right\}$ be subsequences of $\left\{w_{n}\right\}$ and $\left\{u_{n}\right\}$ respectively. Consequently from (40), $w_{n_{j}} \rightarrow \bar{x}$ as $j \rightarrow \infty$. Let $B$ be a mapping defined by

$$
B v= \begin{cases}A v+N_{C}(v), & \text { if } v \in C, \\ \emptyset & \text { if } v \notin C .\end{cases}
$$

By (12), $B$ is maximal monotone and $B^{-1}(0)=V I(C, A)$. If we let $(v, w) \in G(B)$, then $w \in B v=A v+N_{C}(v)$ and thus $w-A v \in N_{C}(v)$. This implies that

$$
\langle v-t, w-A v\rangle \geq 0, \quad \text { for all } t \in C,
$$

and in particular

$$
\left\langle v-w_{n_{j}}, w-A v\right\rangle \geq 0 .
$$

Since $w_{n_{j}}=P_{C}\left(u_{n_{j}}-\mu_{n_{j}} A u_{n_{j}}\right)$, by the characterization of $P_{C}$, we obtain

$$
\left\langle w_{n_{j}}-v, u_{n_{j}}-\mu_{n_{j}} A u_{n_{j}}-w_{n_{j}}\right\rangle \geq 0, \text { for all } v \in C .
$$

Hence

$$
\left\langle v-w_{n_{j}}, \frac{w_{n_{j}}-u_{n_{j}}}{\mu_{n_{j}}}+A u_{n_{j}}\right\rangle \geq 0, \quad \text { for all } \quad v \in C .
$$

Therefore, we have from (45) and (46) that

$$
\begin{aligned}
\left\langle v-w_{n_{j}}, w\right\rangle & \geq\left\langle v-w_{n_{j}}, A v\right\rangle \\
& \geq\left\langle v-w_{n_{j}}, A v\right\rangle-\left\langle v-w_{n_{j}}, \frac{w_{n_{j}}-u_{n_{j}}}{\mu_{n_{j}}}+A u_{n_{j}}\right\rangle \\
& =\left\langle v-w_{n_{j}}, A v-A w_{n_{j}}\right\rangle+\left\langle v-w_{n_{j}}, A w_{n_{j}}-A u_{n_{j}}\right\rangle-\left\langle v-w_{n_{j}}, \frac{w_{n_{j}}-u_{n_{j}}}{\mu_{n_{j}}}\right\rangle \\
& \geq\left\langle v-w_{n_{j}}, A w_{n_{j}}-A u_{n_{j}}\right\rangle-\left\langle v-w_{n_{j}}, \frac{w_{n_{j}}-u_{n_{j}}}{\mu_{n_{j}}}\right\rangle \\
& \geq\left\langle v-w_{n_{j}}, A w_{n_{j}}-A u_{n_{j}}\right\rangle-\left\|v-w_{n_{j}}\right\||| \frac{w_{n_{j}}-u_{n_{j}}}{\mu_{n_{j}}} \| .
\end{aligned}
$$

Passing to the limit in the above inequality in (47) (using the continuity of $A$ and noting that lim inf $\operatorname{in}_{j \rightarrow \infty} \mu_{n_{j}}>$ $0)$, it follows from (38) that

$$
\langle v-\bar{x}, w\rangle \geq 0 \text {. }
$$

Since $B$ is maximal monotone, it follows that $\bar{x} \in B^{-1}(0)$, hence $\bar{x} \in V I(C, A)$. On the other hand, let $\left\{y_{n_{j}}\right\}$ be a subsequence of $\left\{y_{n}\right\}$. Note that $y_{n_{j}} \rightarrow \bar{x}$ as $j \rightarrow \infty$ (by (41)). For each $i=1,2, \ldots, m, I-S_{i}$ are demiclosed at zero, then it follows from (42) that $\bar{x} \in F\left(S_{i}\right)$. This implies that $\bar{x} \in \bigcap_{i=1}^{m} F\left(S_{i}\right)$. Hence

$$
\bar{x} \in V I(C, A) \cap \bigcap_{i=1}^{m} F\left(S_{i}\right) .
$$

Next, we show that $\left\{x_{n}\right\}$ converges strongly to $x^{\star}$, where $x^{\star}=P_{\Gamma}(I-D+\xi f) x^{\star}$ is a unique solution of the variational inequality

$$
\left\langle(D-\xi f) x^{\star}, x^{\star}-x\right\rangle \leq 0, \quad x \in \Gamma .
$$

To do this, we prove that $\lim \sup _{n \rightarrow \infty}\left\langle(D-\xi f) x^{\star}, x^{\star}-x_{n}\right\rangle \leq 0$. Choose a subsequence $\left\{x_{n_{j}}\right\}$ of $\left\{x_{n}\right\}$ such that

$$
\limsup _{j \rightarrow \infty}\left\langle(D-\xi f) x^{\star}, x^{\star}-x_{n}\right\rangle=\lim _{j \rightarrow \infty}\left\langle(D-\xi f) x^{\star}, x^{\star}-x_{n_{j}}\right\rangle .
$$


Since $x_{n_{j}} \rightarrow \bar{x}$, and using (10), we have

$$
\begin{aligned}
\limsup _{j \rightarrow \infty}\left\langle(D-\xi f) x^{*}, x^{\star}-x_{n}\right\rangle & =\lim _{j \rightarrow \infty}\left\langle(D-\xi f) x^{*}, x^{\star}-x_{n_{j}}\right\rangle \\
& =\left\langle(D-\xi f) x^{\star}, x^{\star}-\bar{x}\right\rangle \\
& =\left\langle x^{\star}-(I-(D-\xi f)) x^{\star}, x^{\star}-\bar{x}\right\rangle \leq 0 .
\end{aligned}
$$

Now using Lemma 2.7, Lemma 3.6(i) and (48), we obtain that $\left\|x_{n}-x^{\star}\right\| \rightarrow 0$, which implies that $\left\{x_{n}\right\}$ converges strongly to $x^{\star}$. This concludes Case A.

CASE B: Suppose $\left\{\left\|x_{n}-p\right\|\right\}$ is not monotonically decreasing. Choose some $n_{0}$ large enough and for all $n \geq n_{0}$, we define $\phi: \mathbb{N} \rightarrow \mathbb{N}$ by

$$
\phi(n)=\max \left\{k \in \mathbb{N}: k \leq n: \phi_{k} \leq \phi_{k+1}\right\} .
$$

Clearly, $\phi$ is nondecreasing, where $\phi(n) \rightarrow \infty$ as $n \rightarrow \infty$ and

$$
0 \leq\left\|x_{\phi(n)}-p\right\| \leq\left\|x_{\phi(n)+1}-p\right\|, \quad \text { for all } n \geq n_{0} .
$$

A similar argument as in CASE A, we obtain

$$
\left\|w_{\phi(n)}-u_{\phi(n)}\right\| \rightarrow 0, \quad\left\|v_{\phi(n), i}-y_{\phi(n)}\right\| \rightarrow 0, \quad\left\|x_{\phi(n)+1}-x_{\phi(n)}\right\| \rightarrow 0,
$$

as $n \rightarrow \infty$ and $\Omega_{w}\left(x_{\phi(n)}\right) \subset V I(C, A) \cap \bigcap_{i=1}^{m} F\left(S_{i}\right)$, where $\Omega_{w}\left(x_{\phi(n)}\right)$ is the weak subsequential limit of $\left\{x_{\phi(n)}\right\}$. Also, we have

$$
\limsup _{n \rightarrow \infty}\left\langle(D-\xi f) p, p-x_{\phi(n)}\right\rangle \leq 0 .
$$

Now, from Lemma 3.4(i) we have

$$
\begin{aligned}
\left\|x_{\phi(n)+1}-p\right\|^{2} \leq & \left(1-\frac{2 \delta_{\phi(n)}(\rho-\xi \lambda)}{1-\delta_{\phi(n)} \xi \lambda}\right)\left\|x_{\phi(n)}-p\right\|^{2}+\frac{2 \delta_{\phi(n)}(\rho-\xi \lambda)}{1-\delta_{\phi(n)} \xi \lambda}\left(2\left\langle\xi f(p)-D p, x_{\phi(n)+1}-p\right\rangle+\delta_{\phi(n)} M\right) \\
& +\frac{\alpha_{\phi(n)} M_{2}\left\|x_{\phi(n)}-x_{\phi(n)-1}\right\|}{1-\delta_{\phi(n)} \xi \lambda},
\end{aligned}
$$

for some $M>0$, and where

$$
M_{2}=\sup _{n \geq 1}\left(\left(1-\delta_{\phi(n)} \rho\right)^{2}\left(\left\|x_{\phi(n)}-p\right\|+\left\|x_{\phi(n)-1}-p\right\|\right)+2\left(1-\delta_{\phi(n)} \rho\right)^{2}\left\|x_{\phi(n)}-x_{\phi(n)-1}\right\|\right) .
$$

Since $\left\|x_{\phi(n)}-p\right\|^{2} \leq\left\|x_{\phi(n)+1}-p\right\|^{2}$, then from (50), we obtain

$$
\begin{aligned}
0 \leq & \left(1-\frac{2 \delta_{\phi(n)}(\rho-\xi \lambda)}{1-\delta_{\phi(n)} \xi \lambda}\right)\left\|x_{\phi(n)}-p\right\|^{2}+\frac{2 \delta_{\phi(n)}(\rho-\xi \lambda)}{1-\delta_{\phi(n)} \xi \lambda}\left(2\left\langle\xi f(p)-D p, x_{\phi(n)+1}-p\right\rangle+\delta_{\phi(n)} M\right) \\
& +\frac{\alpha_{\phi(n)} M_{2}\left\|x_{\phi(n)}-x_{\phi(n)-1}\right\|}{1-\delta_{\phi(n)} \xi \lambda}-\left\|x_{\phi(n)}-p\right\|^{2} .
\end{aligned}
$$

Hence

$$
\begin{aligned}
\frac{2 \delta_{\phi(n)}(\rho-\xi \lambda)}{1-\delta_{\phi(n)} \xi \lambda}\left\|x_{\phi(n)}-p\right\|^{2} \leq & \frac{2 \delta_{\phi(n)}(\rho-\xi \lambda)}{1-\delta_{\phi(n)} \xi \lambda}\left(2\left\langle\xi f(p)-D p, x_{\phi(n)+1}-p\right\rangle+\delta_{\phi(n)} M\right) \\
& +\frac{\alpha_{\phi(n)} M_{2}\left\|x_{\phi(n)}-x_{\phi(n)-1}\right\|}{1-\delta_{\phi(n)} \xi \lambda} .
\end{aligned}
$$

Therefore,

$$
\left\|x_{\phi(n)}-p\right\|^{2} \leq 2\left\langle\xi f(p)-D p, x_{\phi(n)+1}-p\right\rangle+\delta_{\phi(n)} M_{4}+\frac{\alpha_{\phi(n)} M_{2}\left\|x_{\phi(n)}-x_{\phi(n)-1}\right\|}{2 \delta_{\phi(n)}(\rho-\xi \lambda)} .
$$


Since $\left\{x_{\phi(n)}\right\}$ is bounded and $\delta_{\phi(n)} \rightarrow 0$, as $n \rightarrow \infty$, then it follows from (49) and Remark 3.4 that

$$
\lim _{n \rightarrow \infty}\left\|x_{\phi(n)}-p\right\|=0 \text {. }
$$

As a consequence, we obtain that for all $n \geq n_{0}$,

$$
0 \leq\left\|x_{n}-p\right\|^{2} \leq \max \left\{\left\|x_{\phi(n)}-p\right\|^{2},\left\|x_{\phi(n)+1}-p\right\|^{2}\right\}=\left\|x_{\phi(n)+1}-p\right\|^{2} .
$$

Hence, $\lim _{n \rightarrow \infty}\left\|x_{n}-p\right\|=0$. This implies that $\left\{x_{n}\right\}$ converges strongly to $p$. This completes the proof.

Recall that the class of quasi-nonexpansive mappings is 0-demicontractive. Thus, we can also obtain the following result for approximating a common solution of the VIP and a finite family of multivalued quasinonexpansive mappings.

Corollary 3.9. Let $C$ be a nonempty, closed and convex subset of a real Hilbert space $H$, and let $A: C \rightarrow H$ be a monotone and L-Lipschitz mapping. For each $i=1,2, \ldots, m$ let $S_{i}: H \rightarrow C B(H)$ be multivalued quasinonexpansive mappings with constant such that $I-S_{i}$ are demiclosed at zero, $S_{i}(p)=\{p\}$ for all $p \in F\left(S_{i}\right)$ and $i=1,2, \ldots, m$. Suppose $\Gamma=V I(C, A) \cap \bigcap_{i=1}^{m} F\left(S_{i}\right) \neq \emptyset$ and let $f: H \rightarrow H$ be a $\lambda$-contraction with constant $\lambda \in(0,1)$ and let $D$ be a bounded operator with coefficient $\rho>0$ such that $0<\xi<\frac{\rho}{\lambda}$. Let $\left\{x_{n}\right\}$ be generated by Algorithm 3.1 in which Assumptions (C1) and (C3) are satisfied. Then the sequence $\left\{x_{n}\right\}$ converges strongly to a point $z$, where $z=P_{\Gamma}(I-D+\xi f)(z)$ is a unique solution of the variational inequality

$$
\langle(D-\xi f) z, z-x\rangle \leq 0, \quad x \in \Gamma .
$$

Remark 3.10.

(i) For suitable starting points, Algorithm 3.1 generates appropriate solutions which approximate the whole solution set $\Gamma$ as guaranteed by Theorem 3.8. This is an interesting property which is different, for example, from the class of Tikhonov-type regularization approaches where the corresponding sequences always converge to the same solution.

(ii) We emphasize here that Algorithm 3.1 does not require a prior estimate of the Lipschitz constant for its strong convergence.

\section{Numerical example}

In this section, we provide examples to compare our inertial viscosity subgradient extragradient Algorithm 3.1 with Algorithms (5) and (6) of Thong and Hieu [27] and, and with iEgA (9) of Dong et al. [40]. All computations are carried out using Matlab version 2017(a) on a HP personal computer.

We start by giving the following example of multivalued $\lambda$-demicontractive mapping given by Jailoka and Suntai in [48]. Let $H=\mathbb{R}$, and for each $i \in \mathbb{N}$ defined $S_{i}: \mathbb{R} \rightarrow 2^{\mathbb{R}}$ by

$$
S_{i}= \begin{cases}{\left[\frac{-(1+2 i) x}{2},-(1+i) x\right],} & x \leq 0, \\ {\left[-(1+i) x, \frac{-(1+2 i) x}{2}\right],} & x>0 .\end{cases}
$$

Then $S_{i}$ is $\lambda_{i}$-demicontractive with $\lambda_{i}=\frac{4 i^{2}+8 i}{4 i^{2}+12 i+9} \in(0,1)$.

Example 4.1. Many problems arising in signal and image processing can be formulated as inverting the equation system

$$
b=B x+e,
$$

where $x \in \mathbb{R}^{N}$ is the unknown original image or data to be recovered, $b \in \mathbb{R}^{M}$ is the vector of noisy observations, $e$ is an additive noise with bounded variance and $B: \mathbb{R}^{N} \rightarrow \mathbb{R}^{M}$ is a bounded linear observation 
Table 1: Comparison of Algorithm 3.1, THSEgM(I) 5 and THSEgM(II) 6 for Example 4.1.

\begin{tabular}{l|lll}
\hline & Time Taken (Sec) & & \\
\hline & Case(i) & Case(ii) & Case(iii) \\
\hline Algorithm 3.1 & 0.0052 & 0.0069 & 0.0096 \\
THSEgM(I) & 0.0155 & 0.0363 & 0.0312 \\
THSEgM(II) & 0.0367 & 0.0363 & 0.0383 \\
\hline
\end{tabular}

operator. In particular, we note that B is typically ill behaved because it models an acquisition process that encounters loss of information. When attempting to find sparse solutions to linear inverse problems of type (55), a successful model is the convex unconstrained minimization problem

$$
\min _{x \in \mathbb{R}^{N}} \frac{1}{2}\|b-B x\|^{2}+v\|x\|_{1},
$$

where $v$ is a postive number, $\|\cdot\|$ is the Euclidean norm and $\|\cdot\|_{1}$ is the $l_{1}$ norm. The aim of the $l_{1}$ term, which is the convex sparsity-promoting penalty, is to make the small component of $x$ become zero. By means of convex analysis, one is able to show that a minimizer to (56) is actually a solution to the LASSO problem

$$
\min _{x \in \mathbb{R}^{N}} \frac{1}{2}\|b-B x\|^{2} \quad \text { subject to }\|x\|_{1}<t,
$$

for any nonnegative real number $t$ (see [49]). It is easy to see that the optimization problem (57) is a special case of the variational inequality problem (1), where $A(x)=B^{T}(B x-b)$ and $C=\left\{x:\|x\|_{1} \leq t\right\}$. Hence, we can use the proposed Algorithm (3.1) to approximate a solution of (55). The projection onto the closed $l_{1}$ ball in $\mathbb{R}^{N}$ is computed through the soft thresholding operator defined by

$$
P_{C}(x)=\mathbb{S}(x)=\operatorname{argmin}_{u \in \mathbb{R}^{N}}\left\{\|x-u\|^{2}+\lambda\|x\|_{1}\right\},
$$

for $\lambda>0$. We set $f(x)=\frac{x}{16}, D(x)=x, \sigma=6, \delta=0.9, \eta=0.7, \delta_{n}=\frac{1}{n+1}, \epsilon_{n}=\frac{1}{(n+1)^{4}}$ and $\alpha=3$ in Algorithm 3.1, and for each $n \in \mathbb{N}$ and $i \geq 0$, define

$$
\beta_{n, i}= \begin{cases}0 & \text { if } n<i, \\ 1-\frac{n}{n+1} \sum_{k=1}^{n} \frac{1}{2^{k}} & \text { if } n=i, \\ \frac{1}{2^{i+1}}\left(\frac{n}{n+1}\right) & \text { if } n>i .\end{cases}
$$

We set the image to go through a random blur and random noise and choose different values of starting point as follows: $x_{0}=-0.5 * \operatorname{randn}(N, 1)$ and $x_{1}=2 \star \operatorname{randn}(N, 1)$, where

$$
\text { Case(i) } N=100, \text { Case(ii) } N=200 \text { and Case(iii) } N=500 \text {. }
$$

We then plot the graphs of the error term $\left(\left\|x_{n+1}-x_{n}\right\|\right)$ against number of iterations for Algorithm 3.1, THSEgM(i) and THSEgM(ii). The numerical result is shown in Table 1 and Figure 1. The stopping criterion used is $\frac{\left\|x_{n+1}-x_{n}\right\|}{\left\|x_{2}-x_{1}\right\|} \leq 10^{-4}$.

Example 4.2. Suppose $H=L^{2}([0,1])$ with $\|x\|_{L^{2}}:=\left(\int_{0}^{1}|x(t)|^{2} d t\right)^{\frac{1}{2}}$ andinner product $\langle x, y\rangle:=\int_{0}^{1} x(t) y(t) d t$, for all $x, y \in H$. Define $A: H \rightarrow H$ by

$$
A(x(t))=\max \{0, x(t)\} .
$$

It is easy to verify that $A$ is 1-Lipschitz continuous and monotone on $H$. Let $C:=\{x \in H:\|x\| \leq 1\}$ be the unit ball. It is known that

$$
P_{C}(x)= \begin{cases}\frac{x}{\|x\|_{L^{2}}} & \text { if }\|x\|_{L^{2}}>1 \\ x & \text { if }\|x\|_{L^{2}} \leq 1\end{cases}
$$



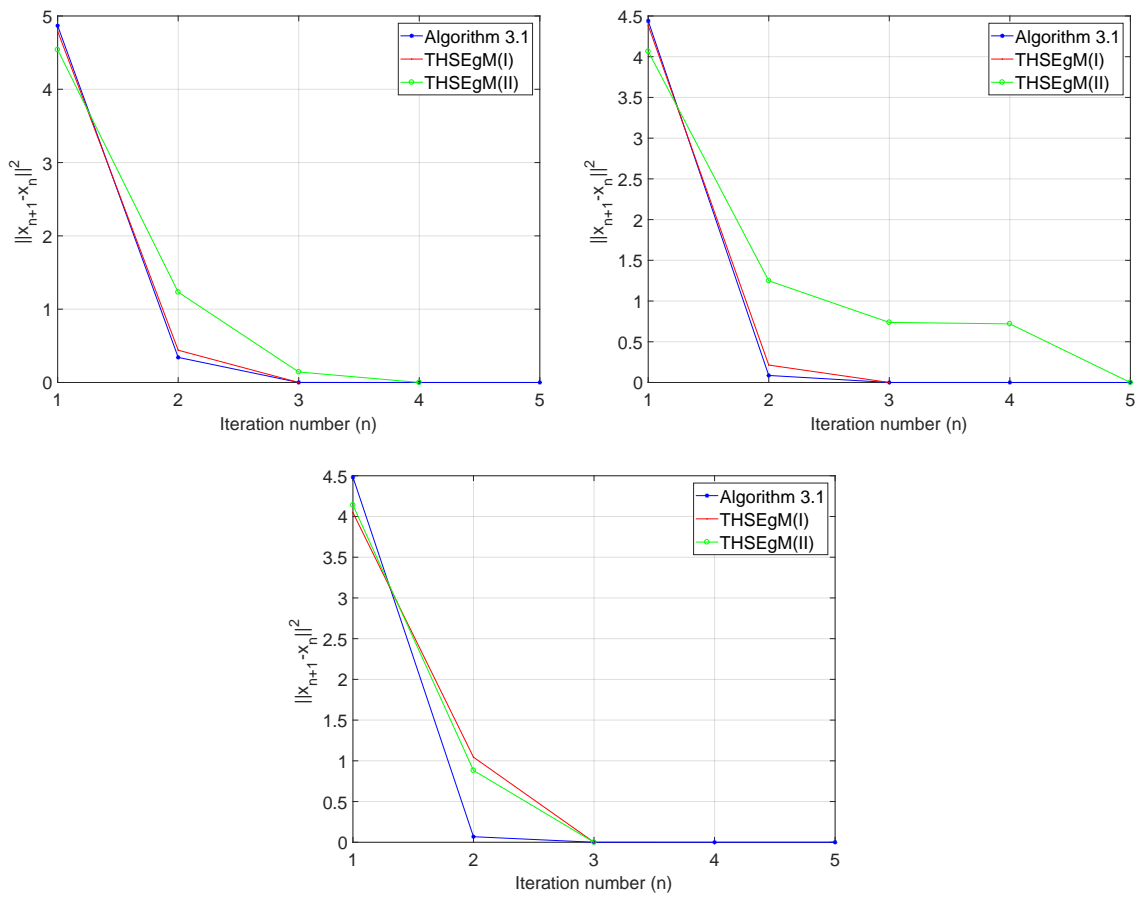

Figure 1: Example 4.1, Top-Left: Case(i); Top-Right: Case(ii); Bottom: Case(iii).

Table 2: Comparison between Algorithm 3.1 and iEgA (9) for Example 4.2.

\begin{tabular}{l|lll}
\hline & Time taken (Secs) & & \\
\hline Algorithms & Case(i) & Case(ii) & Case(iii) \\
\hline Algorithm 3.1 & 0.3641 & 0.1257 & 0.2523 \\
iEgA (9) & 0.5260 & 0.3291 & 1.7566 \\
\hline
\end{tabular}

For $i=1,2, \ldots, m$, let $\left.S_{i}: L^{2}([0,1)]\right) \rightarrow L^{2}([0,1])$ be defined by

$$
\left(S_{i} x\right)(t)=\int_{0}^{1} x(t) d t, \quad t \in[0,1] .
$$

Clearly, $S_{i}$ is 0-demicontractive and $\Gamma=V I(C, A) \cap \bigcap_{i \in \mathbb{N}} F\left(S_{i}\right)=\{0\}$. We choose the following starting points:

Case(i): $\quad x_{0}(t)=t^{2} \exp (7 t)$ and $x_{1}(t)=\frac{1}{6} \sin (-3 t)$,

Case(ii): $\quad x_{0}(t)=0.5 \cos (t)$ and $x_{1}(t)=\cos (-10 t)$,

Case(iii): $\quad x_{0}(t)=5 \exp (t)$ and $x_{1}(t)=\frac{2}{3} \cos (t)$.

Let $f(x)=\frac{x(t)}{2}, D(x)=\int_{0}^{1} x(t) d t, \sigma=4, \eta=0.5, \delta_{n}=\frac{1}{n+1}, \epsilon_{n}=\frac{1}{(n+1)^{2}}, \alpha=3$, and $\beta_{n, i}=\frac{1}{n}$ for each $i \in \mathbb{N} \cup\{0\}$. Using $\frac{\left\|x_{n+1}-x_{n}\right\|}{\left\|x_{2}-x_{1}\right\|}<10^{-6}$ as a stopping criterion, we plot the garphs of $\left\|x_{n+1}-x_{n}\right\|$ against the number of iterations for both Algorithm 3.1 and $\operatorname{iEgA~(9).~The~numerical~result~is~shown~in~Table~} 2$ and Figure 2.

\section{Concluding remarks}

The paper has introduced and proved a strong convergence of a viscosity subgradient extragradient algorithm with inertial extrapolation for approximating common solutions of a variational inequality problem and a 

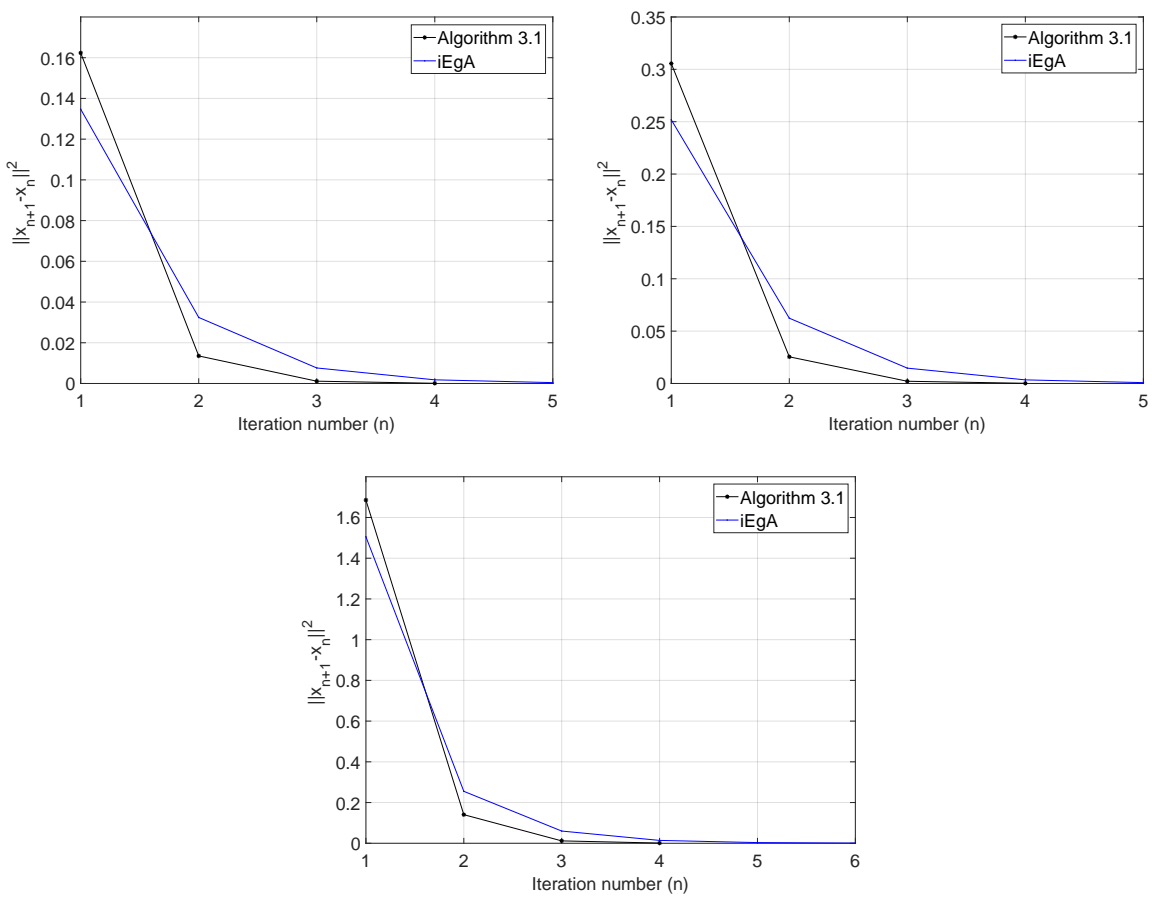

Figure 2: Example 4.2, Top-Left: Case(i); Top-Right: Case(ii); Bottom: Case(iii).

fixed point of a finite family of demicontractive mappings in a real Hilbert space. Some numerical examples are also provided to show that the proposed scheme converges faster than some existing ones. We highlight some further contributions made in this paper as follows:

(i) Unlike the results of Censor et al. [13], Kraikaew and Saejung [50], Thong and Hieu [27] which require that the stepsize satisfies $\mu_{n}=\mu \in\left(0, \frac{1}{L}\right)$, where $L$ is the Lipschitz constant of $A$. In this paper, the stepsize $\mu_{n}$ is determined through a line search which gives room for a larger stepsize value at each iteration. This also improves the corresponding results in [40] and [51].

(ii) Also, the inertial term technique in Step 1 of Algorithm 3.1 is new and efficient. It helps us to avoid imposing some strong conditions that have usually been used for inertial-type algorithms by many authors, see for instance $[33,37,39]$ and reference there in. Furthermore, it assists in obtaining the strong convergence of the sequence $\left\{x_{n}\right\}$ generated by Algorithm 3.1. This improves the weak convergence results of inertial-type algorithms announced by, for instance [33, 40, 52].

(iii) In [53], using a Mann-type algorithm, the authors proved a weak convergence result of an inertial subgradient extragradient algorithm where as, in this paper we used a viscosity approximation method and proved a strong convergence result of an inertial subgradient extragradient algorithm in a real Hilbert space.

\section{References}

[1] Izuchukwu C., Ugwunnadi G. C., Mewomo 0. T., Khan A. R., Abbas M., Proximal-type algorithms for split minimization problem in p-uniformly convex metric space, Numer. Algorithms, 2018, https://doi.org/10.1007/s11075-018-0633-9

[2] Aremu K. O., Izuchukwu C., Ugwunnadi G. C., Mewomo O. T., On the proximal point algorithm and demimetric mappings in CAT(0) spaces, Demonstr. Math., 2018, 51, 277-294

[3] Okeke C. C., Mewomo 0. T., On split equilibrium problem, variational inequality problem and fixed point problem for multivalued mappings, Ann. Acad. Rom. Sci. Ser. Math. Appl., 2017, 9(2), 223-248 
[4] Okeke C. C., Bello A. U., Izuchukwu C., Mewomo 0. T., Split equality for monotone inclusion problem and fixed point problem in real Banach spaces, Aust. J. Math. Anal. Appl., 2017, 14(2), 1-20

[5] Izuchukwu C., Aremu K. O., Mebawondu A. A., Mewomo 0. T., A viscosity iterative technique for equilibrium and fixed point problems in a Hadamard space, Appl. Gen. Topol., 2019, doi:10.4995/agt.2019.10635

[6] Kinderlehrer D., Stampachia G., An introduction to variational inequalities and their applications, Society for Industrial and Applied Mathematics, Philadelphia, 2000

[7] Korpelevich G. M., An extragradient method for finding saddle points and for other problems, Ekon. Mat. Metody, 1976, 12(4), 747-756

[8] Apostol R. Y., Grynenko A. A., Semenov V. V., Iterative algorithms for monotone bilevel variational inequalities, J. Comput. Appl. Math., 2012, 107, 3-14

[9] Ceng L. C., Hadjisavas N., Weng N. C., Strong convergence theorems by a hybrid extragradient-like approximation method for variational inequalities and fixed point problems, J. Glob. Optim., 2010, 46, 635-646

[10] Censor Y., Gibali A., Reich S., Extensions of Korpelevich's extragradient method for variational inequality problems in Euclidean space, Optim., 2012, 61(9), 1119-1132

[11] Denisov S. V., Semenov V. V., Chabak L. M., Convergence of the modified extragradient method for variational inequalities with non-Lipschitz operators, Cybern. Syst. Anal., 2015, 51(5), 757-765

[12] Ogbuisi F. U., Mewomo O. T., Iterative solution of split variational inclusion problem in real Banach space, Afr. Mat., 2017, 28(1-2), 295-309

[13] Censor Y., Gibali A., Reich S., The subgradient extragradient method for solving variational inequalities in Hilbert spaces, J. Optim. Theory Appl., 2011, 148(2), 318-335

[14] Mainge P. E., Gobinddass M. L., Convergence of one-step projected gradient methods for variational inequalities, J. Optim. Theory Appl., 2016, 171, 146-168

[15] Mainge P. E., Numerical approach to monotone variational inequalities by a one-step projected reflected gradient method with the line-search procedure, Comput. Math. Appl., 2016, 72(3), 720-728

[16] Malitsky Y. V., Projected reflected gradient methods for monotone variational inequalities, SIAM J. Optim., 2015, 25, 502520

[17] Bauschke H. H., Combettes P. L., A weak-to-strong convergence principle for Fejér-monotone methods in Hilbert spaces, Math. Oper. Res., 2001, 26(2), 248-264

[18] liduka H., Acceleration method for convex optimization over the fixed point set of a nonexpansive mappings, Math. Prog. Series A., 2015, 149(1-2), 131-165

[19] Jolaoso L. O., Ogbuisi F. U., Mewomo O. T., An iterative method for solving minimization, variational inequality and fixed point problems in reflexive Banach spaces, Adv. Pure Appl. Math., 2018, 9(3), 167-184

[20] Mainge P. E., A hybrid extragradient viscosity method for monotone operators and fixed point problems, SIAM J. Control Optim., 2008, 49, 1499-1515

[21] Mewomo O. T., Ogbuisi F. U., Convergence analysis of an iterative method for solving multiple-set split feasibility problems in certain Banach spaces, Quaest. Math., 2018, 41(1), 129-148

[22] Ogbuisi F. U., Mewomo O. T., On split generalized mixed equilibrium problems and fixed point problems with no prior knowledge of operator norm, J. Fixed Point Theory Appl., 2017, 19(3), 2109-2128

[23] Ogbuisi F. U., Mewomo O. T., Convergence analysis of common solution of certain nonlinear problems, Fixed Point Theory, 2018, 19(1), 335-358

[24] Oyewole K. O., Jolaoso L. O., Izuchuwu C., Mewomo O. T., On approximation of common solution of finite family of mixed equilibrium problems involving $\mu-\eta$ relaxed monotone mapping in a Banach space, Politehn. Univ. Bucharest Sci. Bull. Ser. A Appl. Math. Phys., 2019, 81(1), 19-34

[25] Shehu Y., Mewomo O. T., Further investigation into split common fixed point problem for demicontractive operators, Acta Math. Sin. (Engl. Ser.), 2016, 32(11), 1357-1376

[26] Censor Y., Gibali A., Reich S., Algorithms for the split variational inequalities problems, Numer. Algorithms, 2012, 59, 301323

[27] Thong D. V., Hieu D. V., Modified subgradient extragradient algorithms for variational inequalities problems and fixed point algorithms, Optim., 2018, 67(1), 83-102

[28] Moudafi A. , Viscosity approximation method for fixed-points problems, J. Math. Anal. Appl., 2000, 241(1), 46-55

[29] Xu H. K., Viscosity approximation method for nonexpansive mappings, J. Math. Anal. Appl., 2004, 298(1), 279-291

[30] Polyak B. T., Some methods of speeding up the convergence of iteration methods, U.S.S.R. Comput. Math. Math. Phys., 1964, 4(5), 1-17

[31] Alvarez F., Attouch H., An inertial proximal method for monotone operators via discretization of a nonlinear oscillator with damping, Set-Valued Anal., 2001, 9(1-2), 3-11

[32] Moudafi A., Oliny M., Convergence of a splitting inertial proximal method for monotone operators, J. Comput. Appl. Math., 2003, 155(2), 447-454

[33] Lorenz D., Pock T., An inertial forward-backward algorithm for monotone inclusions, J. Math. Imaging Vision, 2015, 51(2), 311-325 
[34] Jolaoso L. O., Oyewole K. O., Okeke C. C., Mewomo O. T., A unified algorithm for solving split generalized mixed equilibrium problem and fixed point of nonspreading mapping in Hilbert space, Demonstr. Math., 2018, 51, 211-232

[35] Chan R. H., Ma S., Jang J. F., Inertial proximal ADMM for linearly constrained separable convex optimization, SIAM J. Imaging Sci., 2015, 8(4), 2239-2267

[36] Beck A., Teboulle M., A fast iterative shrinkage-thresholding algorithm for linear inverse problem, SIAM J. Imaging Sci., 2009, 2(1), 183-202

[37] Chambolle A., Dossal Ch., On the convergence of the iterates of the "fast iterative shrinkage/thresholding algorithm", J. Optim. Theory Appl., 2015, 166(3), 968-982

[38] Mainge P. E., Convergence theorems for inertial KM-type algorithms, J. Comput. Appl. Math., 2008, 219(1), 223-236

[39] Bot R. I., Csetnek E. R., Hendrich C., Inertial Douglas-Rachford splitting for monotone inclusion problems, Appl. Math. Comput., 2015, 256, 472-487

[40] Dong Q.-L., Lu Y. Y., Yang J., The extragradient algorithm with inertial effects for solving the variational inequality, Optim., 2016, 65(12), 2217-2226

[41] Rockafellar R. T., Monotone operators and the proximal point algorithms, SIAM J. Control Optim., 1976, 14(5), 877-898

[42] Song Y., Cho Y. J., Some note on Ishikawa iteration for multivalued mappings, Bull. Korean Math. Soc., 2011, 48(3), 575-584

[43] Marino G., Xu H. K., Weak and strong convergence theorems for strict pseudo-contraction in Hilbert spaces, J. Math. Anal. Appl., 2007, 329, 336-346

[44] Zegeye H., Shahzad N., Convergence of Mann's type iteration method for generalized asymptotically nonexpansive mappings, Comput. Math. Appl., 2011, 62(11), 4007-4014

[45] Chidume C. E., Ezeora J. N., Krasnoselskii-type algorithm for family of multi-valued strictly pseudo-contractive mappings, Fixed Point Theory Appl., 2014, 2014:111

[46] Xu H. K., Iterative algorithms for nonlinear operators, J. Lond. Math. Soc., 2002, 66, 240-256

[47] Fang C., Chen S., Some extragradient algorithms for variational inequalities, In: Han W., Migórski S., Sofonea M. (Eds.), Advances in Variational and Hemivariational Inequalities, Advances in Mechanics and Mathematics, Springer, Cham, 2015, 33, $145-171$

[48] Jailoka P., Suantai S., The split common fixed point problem for multivalued demicontractive mappings and its applications, RACSAM, 2018, https://doi.org/10.1007/s13398-018-0496-x

[49] Figureido M. A., Norwak R. D., Wright S. J., Gradient projection for sparse reconstruction: Application to compressed sensing and other inverse problem, IEEE J. Sel. Top. Signal Process., 2007, 1, 586-598

[50] Kraikaew R., Saejung S., Strong convergence of the Halpern subgradient extragradient method for solving variational inequalities in Hilbert spaces, J. Optim. Theory Appl., 2014, 163(2), 399-412

[51] Thong D. V., Hieu D. V., Modified subgradient extragradient method for variational inequality problems, Numer. Algor., 2018, 79(2), 597-610

[52] Chuang C. S., Hybrid inertial proximal algorithm for the split variational inclusion problem in Hilbert spaces with applications, Optim., 2017, 66(5), 777-792

[53] Thong D. V., Hieu D. V., Inertial subgradient extragradient algorithms with line-search process for solving variational inequality problems and fixed point problems, Numer. Algor., 2018, https://doi.org/10.1007/s11075-018-0527-x 